OPEN ACCESS

Edited by: Wei Du,

Wilkes University, United States

Reviewed by:

Tatiana S. Perova,

Trinity College Dublin, Ireland

Huiyun Liu,

University College London

United Kingdom

${ }^{*}$ Correspondence:

Jifeng Liu

jifeng.liu@dartmouth.edu

Specialty section:

This article was submitted to

Optics and Photonics,

a section of the journal

Frontiers in Physics

Received: 28 June 2019 Accepted: 03 September 2019

Published: 23 September 2019

Citation:

Wang X, Cuervo Covian A, Je L, Fu S, Li H, Piao J and Liu J (2019) GeSn on Insulators (GeSnOl) Toward Mid-infrared Integrated Photonics. Front. Phys. 7:134

doi: 10.3389/fphy.2019.00134

\section{GeSn on Insulators (GeSnOI) Toward Mid-infrared Integrated Photonics}

\author{
Xiaoxin Wang ${ }^{1}$, Alejandra Cuervo Covian ${ }^{1}$, Lisa Je ${ }^{1}$, Sidan Fu ${ }^{1}$, Haofeng $\mathrm{Li}^{1}$, \\ James Piao ${ }^{1,2}$ and Jifeng Liu ${ }^{1 *}$ \\ ${ }^{1}$ Thayer School of Engineering, Dartmouth College, Hanover, NH, United States, ${ }^{2}$ Epitaxial Laboratory Inc., Syracuse, NY, \\ United States
}

In recent years, $\mathrm{Ge}$ and $\mathrm{Ge}_{1-x} \mathrm{Sn}_{x}$ materials and devices have achieved rapid progress in integrated photonics. However, conventional heteroepitaxy of active photonic devices compromises the area on Si for CMOS electronics, limiting the scale of integration. Furthermore, it is not possible to grow GeSn epitaxially on amorphous and/or flexible substrates toward 3D photonic integration in mid-infrared (MIR) regime. Here, we present low-temperature crystallization of direct bandgap, high crystallinity $\mathrm{Ge}_{1-\mathrm{x}} \mathrm{Sn}_{\mathrm{x}}(0.08<\mathrm{x}<$ 0.26) on amorphous dielectrics insulators (GeSnOI) toward 3D and flexible MIR integrated photonics. Utilizing eutectically-enhanced crystallization (EEC), an extraordinarily large average grain size of $\sim 100 \mu \mathrm{m}$ has been achieved in blanket GeSn films crystallized on $\mathrm{SiO}_{2}$ layers, flexible glass, and polyimide substrates alike. Furthermore, using $\mathrm{Sn}$ nanodot enhanced composition enhancement (NICE), we have achieved an average Sn composition as high as 26 at.\% to further extend the optical response of GeSn toward $\lambda$ $=3-5 \mu \mathrm{m}$. The achieved Sn composition of 8-26 at.\% far exceeds that of the equilibrium solubility limit of $<1$ at.\%, even though the crystallization temperature of $350-450^{\circ} \mathrm{C}$ far exceeds the typical epitaxial growth temperature of GeSn. This result indicates that crystallization from amorphous GeSn (a-GeSn) may offer better metastability compared to direct epitaxial growth of GeSn. Attesting to the high crystallinity, a peak optical gain of 2,900 $\mathrm{cm}^{-1}$ with a lifetime approaching $0.1 \mathrm{~ns}$ is achieved at $\lambda=2,200-2,350 \mathrm{~nm}$ at $300 \mathrm{~K}$. The gain lifetime is on the same order as epitaxial GeSn, and it is $>100 x$ longer than the direct gap transition in Ge, confirming the indirect-to-direct band gap transition in GeSn at $\sim 9$ at. Sn composition. Moreover, a prototype p-GeSn/n-Si photodiode from a-GeSn crystallization achieves $100 \mathrm{~mA} / \mathrm{W}$ responsivity at $\lambda=2,050 \mathrm{~nm}$ and $\mathrm{T}=300 \mathrm{~K}$, approaching the level of some commercial PbS detectors. The device also demonstrates photovoltaic behavior and a low dark current density of $1 \mathrm{~mA} / \mathrm{cm}^{2}$ at $-1 \mathrm{~V}$ reverse bias, comparable to epitaxial $\mathrm{Ge} / \mathrm{Si}$ photodiodes. These results indicate that crystallization of GeSnOl offers a promising solution for active devices toward 3D MIR photonic integration and/or MIR photonics on flexible substrates.

Keywords: GeSn, direct band gap, crystallization, photonic integration, mid-infrared, optical gain, photodetector 


\section{INTRODUCTION}

Integrated photonics has achieved rapid progress in the past two decades. An almost complete set of passive and active photonic components have been developed on the silicon-on-insulator (SOI) platform, including waveguides, couplers, splitters, filters, polarizers, (de)multiplexers, modulators, switches, and photodetectors [1, 2]. Many of these devices are being standardized in Silicon Photonics Process Design Kits (PDK) under the American Institute for Manufacturing Photonics (AIM Photonics $)^{1}$. Ge has played an important role in active photonic devices for integrated photonics [3-5] due to its pseudo direct bandgap behavior [6] and compatibility with Si complementary metal oxide semiconductor (CMOS) technology. More recently, $\mathrm{Ge}_{1-\mathrm{x}} \mathrm{Sn}_{\mathrm{x}}$ materials [7-13] and devices [14-20] have become an active field of research since an indirect-to-direct bandgap transition occurs at $\mathrm{x} \sim 0.06-0.11$ (depending on the level of strain). GeSn also brings about a great opportunity for integrated active photonic devices in mid-infrared (MIR) regime $(\lambda=$ $3-5 \mu \mathrm{m})[21-24]$ for sensing and imaging applications since the direct bandgap can be decreased to $<0.5 \mathrm{eV}$ for $\mathrm{x}>0.09$.

So far, most of the Si-based integrated photonic devices, especially $\mathrm{Ge}$ and $\mathrm{GeSn}$ active devices, are achieved via planar electronic-photonic integration, where photonic components are integrated with CMOS circuitry on the same single-crystal Si layer (Figure 1a). However, heteroepitaxy of Ge and GeSn consumes real estate on single crystal $\mathrm{Si}$, thereby significantly sacrificing CMOS area for photonic devices. This issue is manifested by the scanning electron microscopy (SEM) and transmission electron microscopy (TEM) images in the inset of Figure 1a, showing that the cross-sectional dimensions

$\overline{{ }^{1} \text { http://www.aimphotonics.com/pdk }}$ of a waveguide-integrated Ge photodetector is $\sim 20 \times$ bigger than CMOS transistor in $32 \mathrm{~nm}$ technology node due to the fundamental optical diffraction limit. Moreover, the planar integration approach has to introduce some modifications to existing CMOS process flow in order to accommodate the epitaxial growth as well as the vertical dimension of photonic devices [25], making it hard to implement in $<45 \mathrm{~nm}$ CMOS technology nodes. In addition, it is not possible to epitaxially grow $\mathrm{Ge}$ or $\mathrm{GeSn}$ on amorphous substrates (such as polymers) for flexible integrated photonic circuits, either. Therefore, in large-scale electronic-photonic integration it would be ideal to adopt a monolithic 3D integration approach, moving the photonic circuits to the metal/dielectric interconnect level above the CMOS layer (Figure 1b). The ability to achieve high crystallinity Ge or GeSn on amorphous dielectrics would offer great flexibility in photonic integration since active photonic devices could be grown on any layer or substrate at any designated region.

In this paper, we present low-temperature crystallization of direct bandgap, high crystallinity $\mathrm{Ge}_{1-\mathrm{x}} \mathrm{Sn}_{\mathrm{x}}$ with $\mathrm{x}$ up to 0.26 (i.e., 26 at.\%) on amorphous dielectrics and flexible substrates toward monolithic 3D photonic integration and flexible MIR photonics. Utilizing eutectically enhanced crystallization (EEC) in Ge-Sn system, a very low nucleation rate and high lateral growth rate can be achieved, leading to an extraordinarily large grain size of $\sim 100 \mu \mathrm{m}$ in blanket GeSn films crystallized on thermally grown $\mathrm{SiO}_{2}$ layers, flexible Willow glass, fused silica, and polyimide substrates alike. The crystallization temperature range of $380-450^{\circ} \mathrm{C}$ is also compatible with back-end-of-line CMOS processing and polyimide flexible substrates. Furthermore, using Sn nanodot induced composition enhancement (NICE), we have achieved an average $\mathrm{Sn}$ composition as high as 26 at.\%. Attesting

\section{Monolithic Electronic-Photonic Integration}

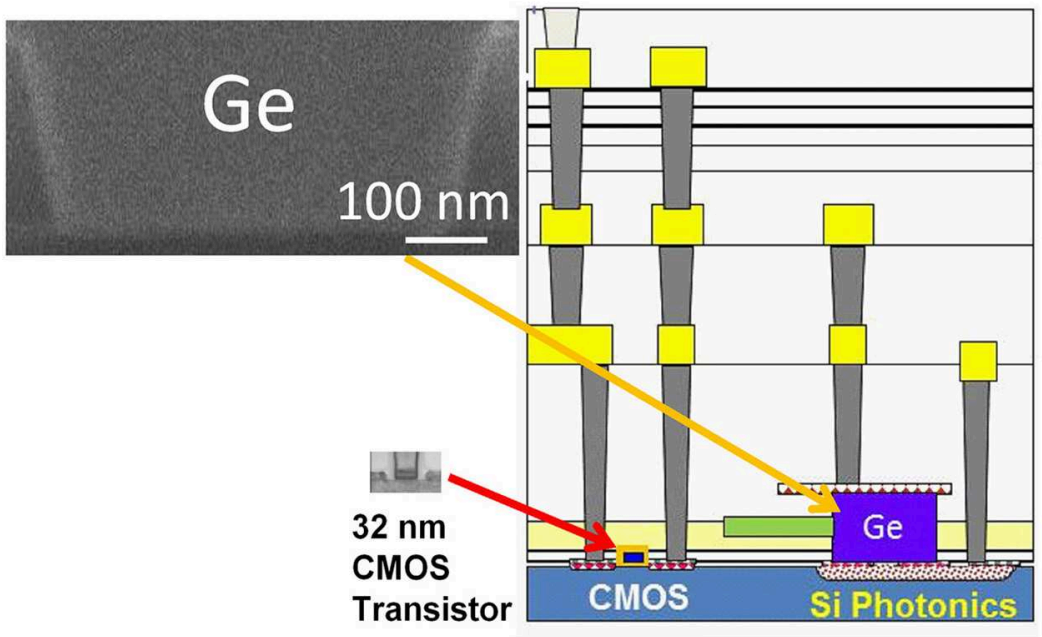

(a)

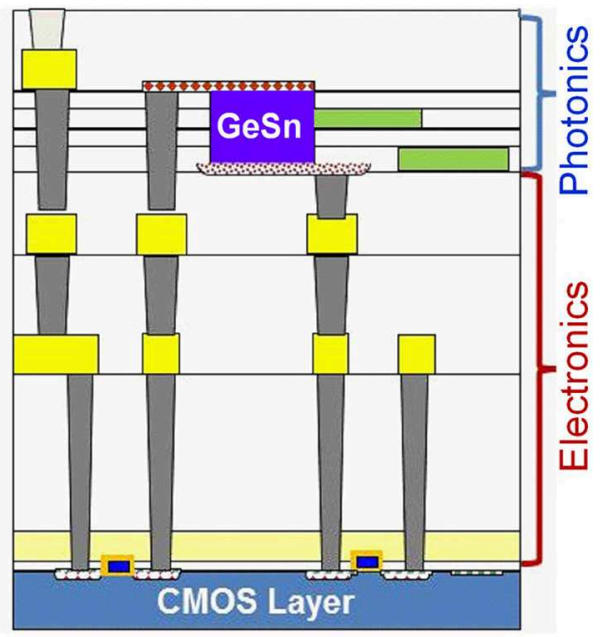

(b)

FIGURE 1 | Schematics of monolithic electronic-photonic integration: (a) Planar integration of photonics with CMOS electronics on the same Si layer, and (b) 3D photonic integration with the photonic interconnect layer positioned well above the CMOS layer. 
to the high material quality of crystallized GeSn, a high optical gain $\sim 2,900 \mathrm{~cm}^{-1}$ with a lifetime of $0.1 \mathrm{~ns}$ have been achieve at $\lambda=2,200-2,350 \mathrm{~nm}$ at room temperature from crystallized GeSn with 9 at.\% Sn. The peak gain coefficient is comparable to III-V semiconductors. The gain lifetime is on the same order as epitaxial GeSn, and it is $>100 x$ longer than that of Ge, confirming the indirect-to-direct band gap transition in $\mathrm{Ge}_{1-\mathrm{x}} \mathrm{Sn}_{\mathrm{x}}$ at $\mathrm{x} \geq$ 0.09 . Furthermore, a prototype $\mathrm{p}-\mathrm{GeSn} / \mathrm{n}$-Si photodiode achieves $100 \mathrm{~mA} / \mathrm{W}$ responsivity at $\lambda=2,050 \mathrm{~nm}$ and $300 \mathrm{~K}$, comparable to some $\mathrm{PbS}$ MIR photodetectors. The GeSn/Si photodiode also demonstrates photovoltaic behavior and a low dark current density of $1 \mathrm{~mA} / \mathrm{cm}^{2}$ at $-1 \mathrm{~V}$ reverse bias, comparable to epitaxial $\mathrm{Ge} / \mathrm{Si}$ photodiodes. These results indicate that GeSn crystallized on dielectrics offers a promising solution for active devices in $3 \mathrm{D}$ photonic integration.

\section{SUBSTRATE-INDEPENDENT GeSn CRYSTALLIZATION ON AMORPHOUS INSULATORS}

The basic idea of crystallizing amorphous GeSn at low temperatures originates from the eutectic phase diagram of the Ge-Sn system [3, 10]. According to the equilibrium phase diagram, a Ge-rich solid phase is in equilibrium with a Snrich liquid phase at temperatures $>231^{\circ} \mathrm{C}$. While the exact temperature and solubility no longer applies to the crystallization of amorphous GeSn (a-GeSn) since it is not in thermodynamic equilibrium, the formation of a small amount of liquid Sn is indeed observed. During the crystallization process of aGeSn thin films upon thermal annealing, which involves rearrangement of atoms into a long-range ordered diamond cubic structure, the Sn-rich liquid phase greatly enhances atomic diffusion, and subsequently, the lateral growth of the Ge-rich GeSn solid phase. Hence, crystallization of a-GeSn is expected to occur at a relatively low temperature with a fast lateral growth rate. This process is somewhat similar to liquid phase epitaxy except that there is no single crystal template. We call it "eutectically-enhanced crystallization" (EEC). Note that a dramatic difference between the crystallization of a-GeSn and the solidification of liquid-phase GeSn is that the incorporation of $\mathrm{Sn}$ into the Ge-rich solid phase could far exceed the equilibrium solubility $(\sim 1$ at. $\%)$ in the former case because the initial state of a-GeSn is far from equilibrium. Furthermore, because of the lower surface energy of Sn compared to Ge, the Sn-rich liquid phase would segregate at the grain boundaries and on the surface of crystallized GeSn. Subsequently, the excess Sn can be easily and selectively etched away using $\mathrm{HCl}$ solution, leaving the crystallized GeSn for integrated photonic device fabrication $[3,10,21,26-29]$.

\section{Blanket GeSn Thin Film Crystallization}

To investigate the crystallization of GeSn thin films, a- $\mathrm{Ge}_{1-\mathrm{x}} \mathrm{Sn}_{\mathrm{x}}$ $(0<\mathrm{x}<0.20)$ thin films are deposited on thermally grown $\mathrm{SiO}_{2}$ (5-10 nm thick) on Si, Corning's flexible Willow glass substrates [30], fused silica, and polyimide foils by co-evaporating $\mathrm{Ge}$ and $\mathrm{Sn}$ in a Kurt Lesker Lab 18 Physical Vapor Deposition
(PVD) machine. The base vacuum of the system is better than $5 \times 10^{-8}$ Torr before the co-evaporation process. Over the course of our studies, the purity of Sn evaporation sources has been improved from $99.999 \%$ ( $5 \mathrm{Ns}$ ) to $99.99999 \%$ (7Ns), while semiconductor grade Ge has been used in all studies. The deposition rate of Ge is typically $0.08 \mathrm{~nm} / \mathrm{s}$, and the Sn deposition rate is adjusted to achieve different a-GeSn compositions. The deposited a-GeSn thin films are annealed at $380-450^{\circ} \mathrm{C}$ in $\mathrm{N}_{2}$ for crystallization. Alternatively, the samples can be crystallized by rapid thermal annealing (RTA) or locally crystallized by a focused laser beam followed by furnace annealing. The excess $\mathrm{Sn}$ after the crystallization is etched away by $37.2 \% \mathrm{HCl}: \mathrm{H}_{2} \mathrm{O}$ solution.

Irrespective of the substrate, we found the following common features of a-GeSn crystallization: (1) a high lateral growth rate vs. a very low nucleation rate, leading to an extraordinarily large average grain size of $\sim 100 \mu \mathrm{m}$ when crystallizing blanket $\mathrm{Ge}_{1-\mathrm{x}} \mathrm{Sn}_{\mathrm{x}}$ films with $0.08<\mathrm{x}<0.15$. (2) Well-defined crystallization temperature resembling thermodynamic phase transition. This feature is drastically different from the kineticsdominated crystallization of amorphous Si. The crystallization temperature of a-GeSn decreases with the increase of $\mathrm{Sn}$ composition [10], and no crystallization will occur even at $5^{\circ} \mathrm{C}$ below the crystallization temperature after prolonged annealing for several hours. Once above the crystallization temperature, on the other hand, the crystallization can be completed within $90 \mathrm{~s}$ in RTA. This phenomenon is in line with the well-defined eutectic temperature in the Ge-Sn equilibrium phase diagram, although the actual crystallization temperature of a-GeSn is beyond the description of the phase diagram due to its nonequilibrium nature. (3) A strong (111) preferred orientation due to minimization of surface energy on amorphous layers [10, 21, 26, 27]. As will be discussed later, this preferred (111) orientation also has a beneficial effect on the strain engineering of GeSn toward direct gap semiconductors.

Figure 2a shows a top-view SEM image of a crystallized $\mathrm{Ge}_{0.913} \mathrm{Sn}_{0.087}$ film on $5 \mathrm{~nm}$-thick thermally grown $\mathrm{SiO}_{2}$ on Si. Grain boundaries can be clearly observed in the low magnification SEM image in Figure 2a, with huge grain sizes on the order of $0.1-1 \mathrm{~mm}$, or 3-4 orders larger than the GeSn thin film thickness (316 $\mathrm{nm}$ in this case, as revealed in Figure $2 \mathbf{b}$ ). This is an extraordinary result for semiconductor thin film crystallization, where typically the grain size is on the same order as the thickness of the thin film [31]. This observation indicates a slow nucleation rate vs. an extremely fast lateral growth rate during GeSn crystallization induced by the EEC mechanism. Electron backscatter diffraction (EBSD) analyses show that each grain predominantly consists of micro-twins as a result of lateral growth from the same nucleation center upon crystallization [10, 26, 27]. Figures $\mathbf{2 b , c}$ show the crosssectional SEM image and the corresponding energy dispersion $\mathrm{X}$-ray spectroscopy (EDS) mapping for compositional analyses, respectively. Ge and $\mathrm{Sn}$ are both distributed uniformly in the films without any $\mathrm{Sn}$ segregation. The Sn composition from the EDS mapping analysis is $8.7 \pm 0.2$ at.\%. The biaxial tensile strain is determined to be $\sim 0.2 \%$ by combining $\mathrm{X}$ ray diffraction (XRD) with Raman spectroscopy analyses, as detailed in [21]. Furthermore, the strong (111) texture of the 

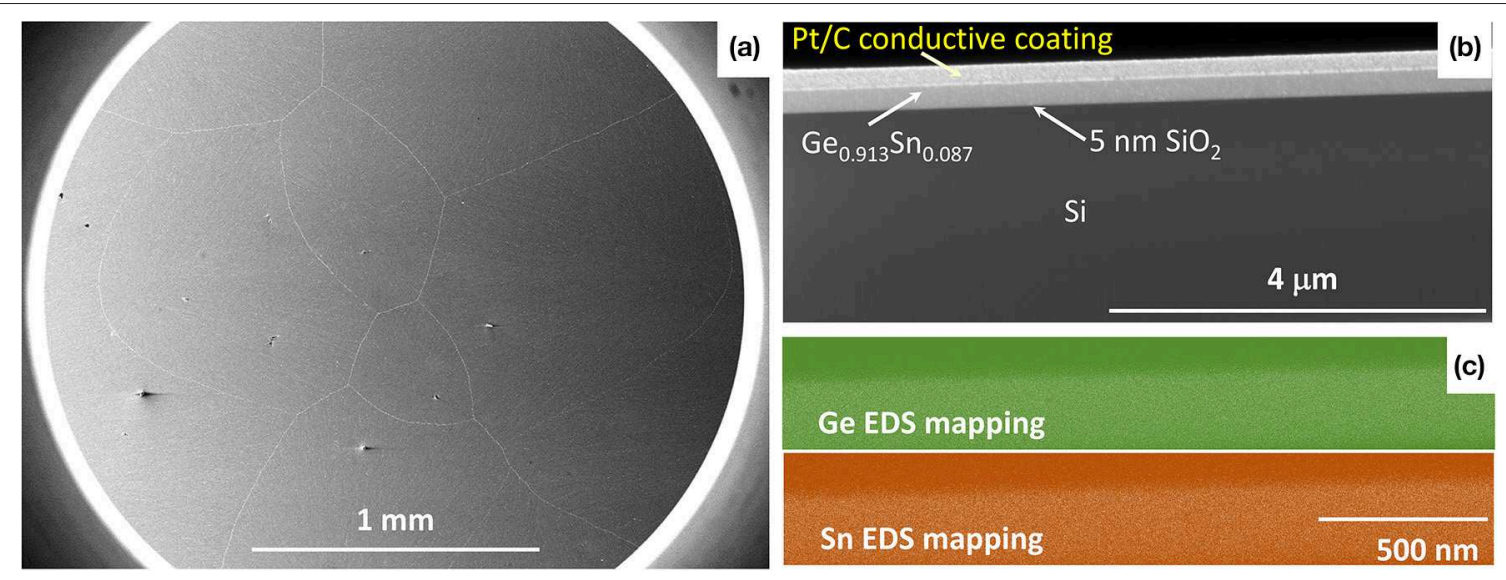

FIGURE 2 | (a) Low-magnification, top-view SEM image of a crystallized $\mathrm{Ge}_{0.913} \mathrm{Sn}_{0.087}$ thin film, showing a large average grain size on the order of $\mathrm{mm}$ scale. (b) Cross-sectional SEM image of the $\mathrm{Ge}_{0.913} \mathrm{Sn}_{0.087}$ film crystallized on thermally-grown $5 \mathrm{~nm}$-thick $\mathrm{SiO}_{2}$ on $\mathrm{Si}$. The film thickness of GeSn is $316 \mathrm{~nm}$. (c) The distribution of Ge and Sn atoms in the GeSn film in (b), as revealed by EDS mapping. Sn is evenly distributed in the film and no Sn clusters observed in the GeSn film.
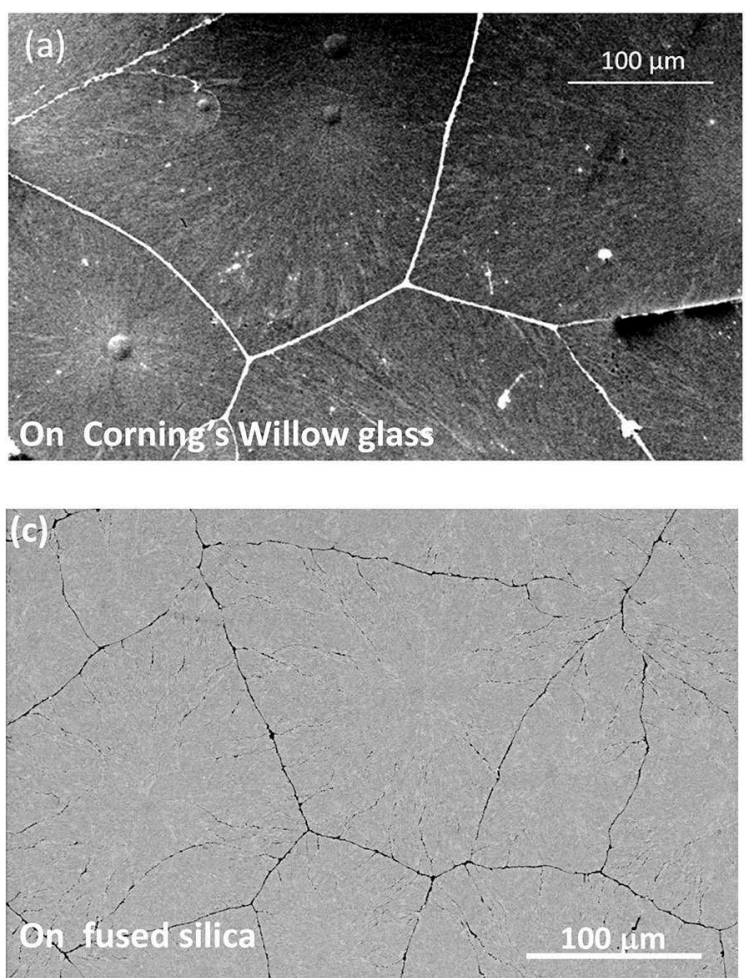
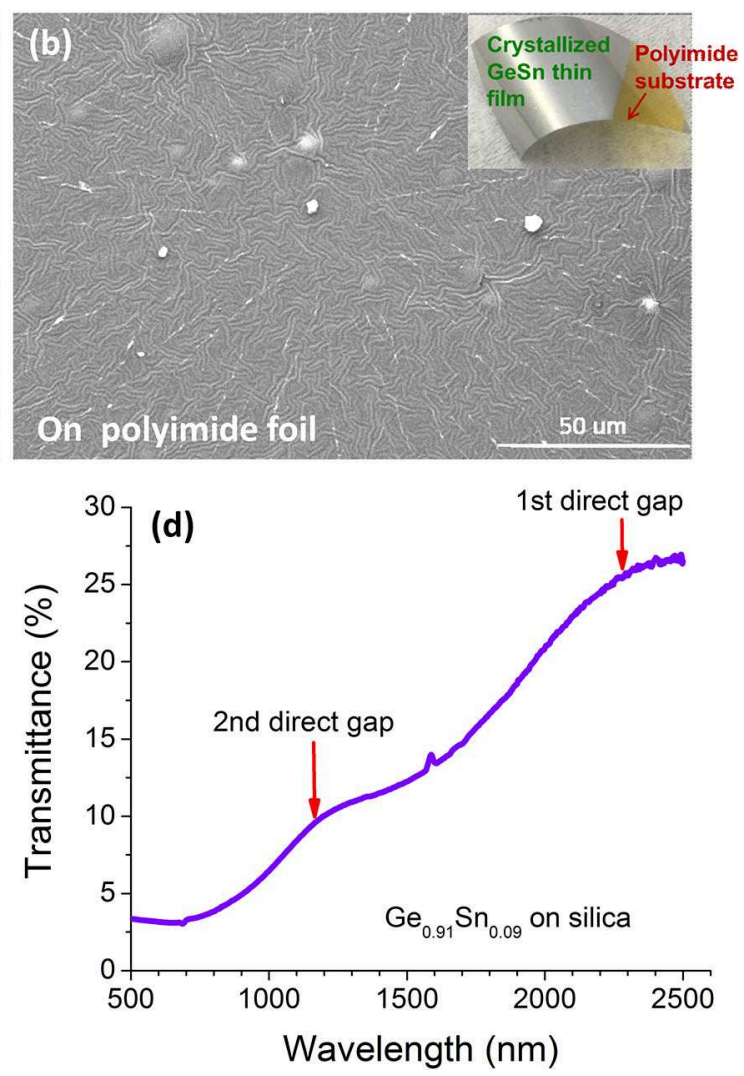

FIGURE 3 | SEM images of Ge $\mathrm{S}_{0.91} \mathrm{Sn}_{0.09}$ ( $300 \mathrm{~nm}$ thick) crystallized on (a) flexible Willow glass substrates; (b) polyimide foils; and (c) fused silica substrate. The inset of (b) shows a photo of the corresponding sample. Panel (d) shows the transmittance spectrum of (c), where the 1st direct gap at $\lambda \sim 2,400$ nm and the 2 nd direct gap at $\lambda \sim 1,150 \mathrm{~nm}$ are clearly observed.

crystallized GeSn thin films leads to a relatively low Poisson's ratio and a higher dilatational strain under biaxial tensile stress due to the largest inter-planar spacing between (111) planes [21]. These factors favor indirect-to-direct gap transition
[21], in contrast to compressive strain in their [001] oriented epitaxial counterparts.

Figure 3 further demonstrates that the EEC mechanism of a-GeSn is equally applicable to flexible Willow glass substrate, 

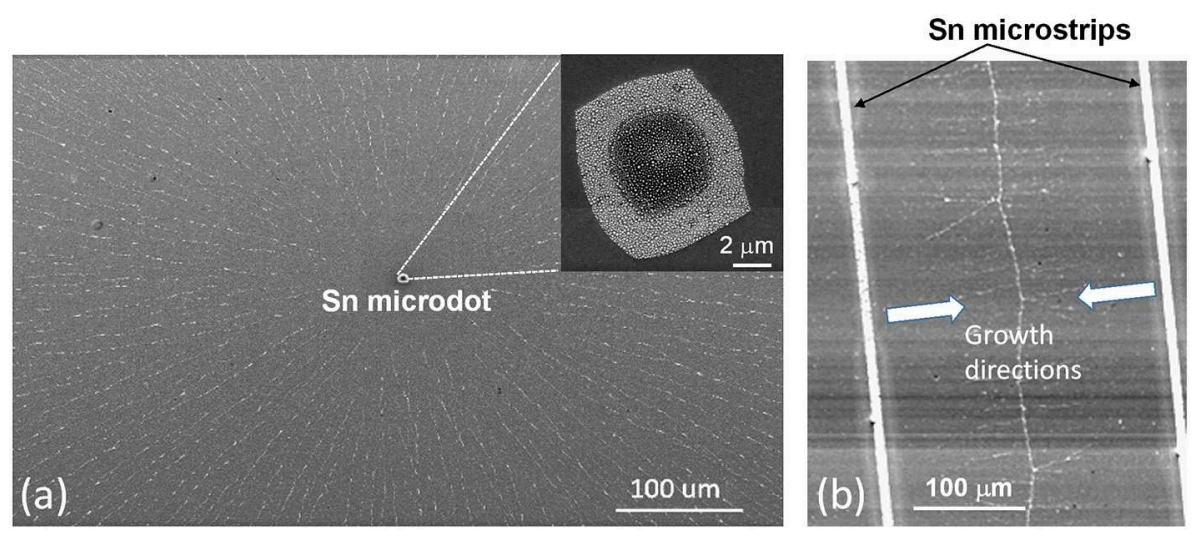

FIGURE 4 | SEM images of $\mathrm{Ge}_{0.91} \mathrm{Sn}_{0.09}$ crystallization induced by Sn micropatterns: (a) nucleation site defined by a Sn microdot (b) nucleation and lateral growth induced by Sn microstrips.

polyimide substrate, and fused silica substrate alike [26, 32]. Large grain sizes of $0.1-1 \mathrm{~mm}$ are maintained in all these cases for $\mathrm{Ge}_{0.91} \mathrm{Sn}_{0.09}$ thin films. In Figure $\mathbf{3 b}$, the center of nucleation can also be clearly seen, from which the grain grows radially. The polyimide substrate also shows some micro wrinkles upon GeSn crystallization due to thermal stress. These wrinkles are similar to surface texturing used in solar cells and can potentially be engineered to enhance the absorption in GeSn. Furthermore, while it is impossible to directly measure the transmittance spectrum of epitaxial GeSn thin films on $\mathrm{Si}$ at $\lambda<1,200 \mathrm{~nm}$ due to the absorption of the Si substrate, the $\mathrm{Ge}_{0.91} \mathrm{Sn}_{0.09}$ thin films crystallized on transparent fused silica substrates reveals both the fundamental direct gap at $\lambda \sim 2,400 \mathrm{~nm}$ and the secondary direct gap at $\lambda \sim 1,150 \mathrm{~nm}$ in the transmittance spectrum (Figure $3 \mathbf{d}$ ). Interestingly, in this case the second direct gap is almost exactly twice the first direct gap, which could make a significant impact on the performance of GeSn lasers due to up-conversion from the first to the second conduction valley through Auger process. These results not only demonstrate substrate-independent, high quality GeSn crystallization, but also show that such studies could offer more information about the band structure of GeSn inaccessible to their epitaxial counterparts. We will come to this point again in section Optical Gain Dynamics of Crystallized Direct Gap GeSnOI.

\section{Controlling the Nucleation Location Upon GeSn Thin Film Crystallization}

While large grains with high crystallinity are achieved in crystallizing blanket $\mathrm{GeSn}$ thin films, the nucleation remains a stochastic process and there is no control of the nucleation site. To pre-define the nucleation sites before crystallization annealing, we have developed three approaches: (1) Sn micropattern induced nucleation [32]; (2) laser seeded nucleation [26-28]; and (3) nanotip induced nucleation [26, 27].

Figures 4a,b show examples of $\mathrm{Sn}$ micropattern induced nucleation. In Figure $4 \mathbf{a}, \mathrm{a} \sim 5 \mu \mathrm{m}$ diameter $\mathrm{Sn}$ microdot is defined on top of a-GeSn by photolithography followed by crystallization annealing. Clearly, the $\mathrm{Sn}$ microdot serves as a nucleation center from which the $\mathrm{Ge}_{0.91} \mathrm{Sn}_{0.09}$ crystals grow radially. The Sn microdot also melts and dewets into smaller Sn nanodots upon crystallization annealing, as shown in the inset of Figure 4a. As discussed earlier, Sn can be removed selectively using $\mathrm{HCl}$ solution after crystallization, offering a convenient approach to define the nucleation sites on GeSn. Figure $4 \mathbf{b}$ further shows two Sn microstrips patterned on top of a-GeSn followed by crystallization annealing. Both $\mathrm{Sn}$ microstrips serve as nucleation centers for GeSn. Therefore, the GeSn grains grow perpendicular to the two $\mathrm{Sn}$ microstrips, respectively, and impinge each other almost exactly in the middle of the two Sn microstrips. This observation further confirms that Sn micropatterns on top of a-GeSn can be engineered to effectively control the nucleation and lateral growth of crystalline GeSn.

An example of laser-seeded crystallization of $\mathrm{Ge}_{0.89} \mathrm{Sn}_{0.11}$ is shown in Figures 5a,b [27]. A laser beam at $\lambda=514 \mathrm{~nm}$ is scanned at one end of a $2 \times 38 \mu \mathrm{m}$ rectangular a-GeSn strip to seed the nucleation of GeSn before furnace annealing. The optical power at the sample surface is measured to be 2-3 $\mathrm{mW}$ during the laser annealing, and the focal spot is $\sim 300 \mathrm{~nm}$ in diameter using a $100 \times$ objective lens. The scan rate is $\sim 1 \mu \mathrm{m} / \mathrm{s}$. Subsequently, the a-GeSn microstrip with a laser seeded nucleation region on one end is annealed at $440^{\circ} \mathrm{C}$ for $30 \mathrm{~min}$. Figure $5 \mathbf{b}$ shows the EBSD mapping of the crystallized strip. Two twinning grains are formed at first from the nucleation site seeded by laser annealing. During the lateral crystal growth along the length direction upon furnace annealing, one of the seed grains (pink) overgrows the other (blue) and becomes dominant. Remarkably, this grain spans almost the entire strip length of $38 \mu \mathrm{m}$, large enough for active photonic devices such as waveguide-coupled modulators or photodetectors. There are a few sporadic twin boundaries inside the microstrip, which are optoelectronically benign since these are coherent interfaces without dangling bonds to induce deeplevel defect centers.

Figures $\mathbf{5 c}$,d show an example of nanotip induced nucleation $[26,27]$. When there is a high curvature surface, such as a nanotip on an a-GeSn micropattern (e.g., the nanotip on the left side of Figure 5c), the local phase transition/crystallization 

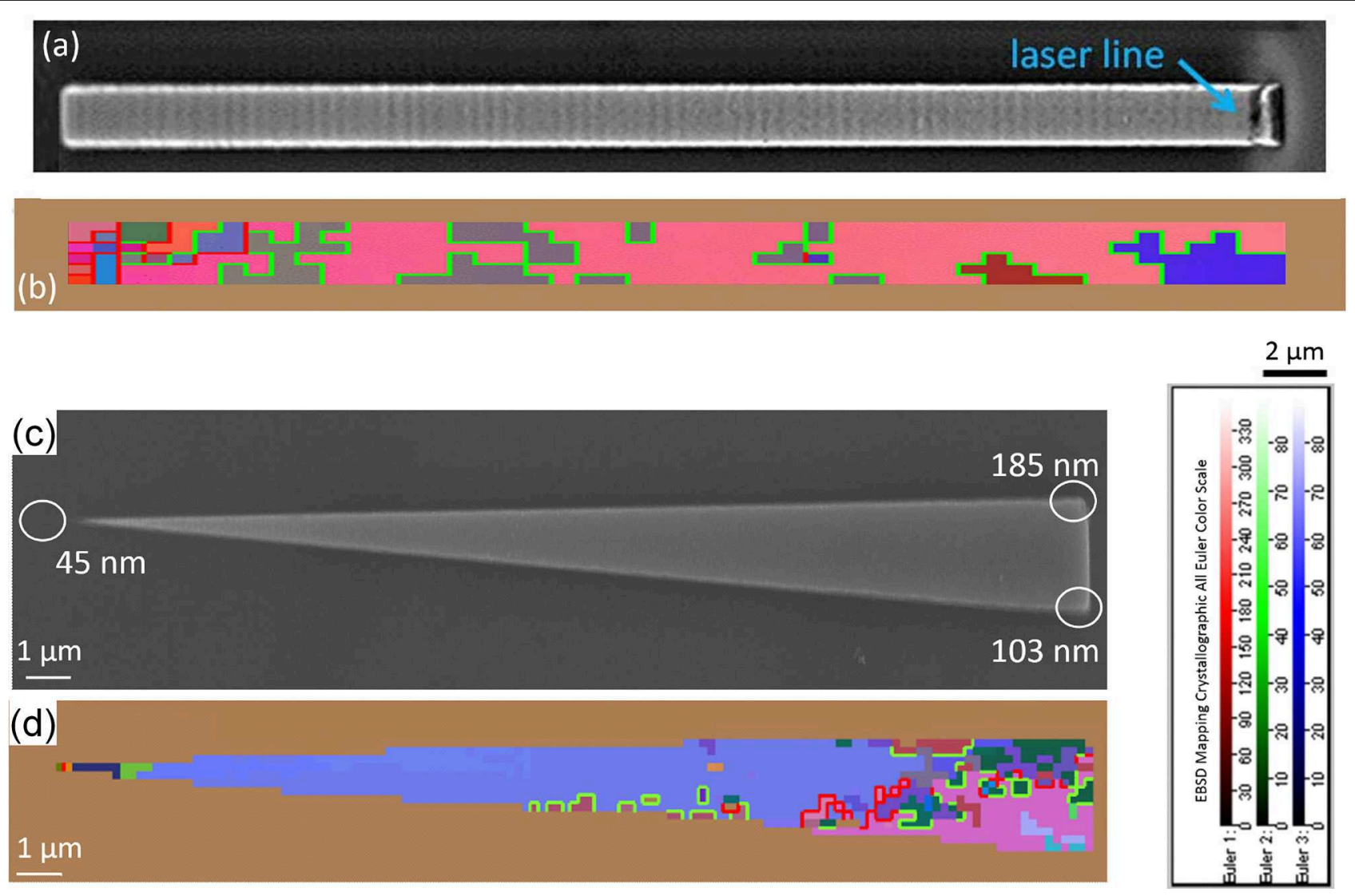

FIGURE 5 | (a) SEM image of a $2 \times 38 \mu \mathrm{m} \mathrm{Ge}_{0.89} \mathrm{Sn}_{0.11}$ microstrip laser annealed at one end (right hand side). The laser scanned along the direction of the width to form a seed for lateral crystal growth. (b) EBSD mapping of the strip after laser seeding and furnace annealing at $440^{\circ} \mathrm{C}$ for $\mathrm{crystallization}$. A dominant grain (in pink) $>35 \mu \mathrm{m}$ long is clearly demonstrated. The twin boundaries are labeled in green. Grain boundaries with misorientation angles $>15^{\circ}$ are labeled in red. The color scale of the Euler angles for the EBSD mapping is also shown in the lower right corner. (c) SEM image of a trianglar $\mathrm{Ge}_{0.885} \mathrm{Sn}_{0.115}$ nanotaper pattern with tip radii on 10-150 nm nanometer scale. The radius of each tip/corner is labeled on the image. (d) Shows the corresponding EBSD mapping of (c) after crystallization. The nanotip on the left has a significantly smaller radius than the other two corners, leading to the growth of a large single crystal grain from this sharpest nanotip.

temperature decreases with the tip radius $r$ as described by the Gibbs-Thomson Equation:

$$
T_{m}(r)=T_{m}(\infty)-\frac{2 T_{m}(\infty) \sigma_{s l}}{\Delta H_{f}(\infty) \rho_{s} r}
$$

Here $T_{m}(r), T_{m}(\infty), \sigma_{s l}, H_{f}(\infty)$, and $\rho_{s}$ are the nanotip crystallization temperature, bulk material crystallization temperature (i.e., $r=\infty$ ), solid/Sn liquid interfacial energy, heat of fusion of the phase transition, and the atomic density of the solid phase, respectively. Therefore, if we anneal an a-GeSn micropattern with a high curvature nanotip at a temperature between $T_{m}(r)$ and $T_{m}(\infty)$, only the nanotip region will crystallize to form a single nucleus. This nucleus at the nanotip will subsequently seed the lateral growth of GeSn across the entire micropattern, leading to a piece of single crystal material. In Figure 5c, a GeSn microtaper pattern with different nanotip curvatures ( $r=45,103$, and $185 \mathrm{~nm}$, respectively) is annealed at $420^{\circ} \mathrm{C}$ for $30 \mathrm{~min}$. Since the tip on the left has a much smaller radius than the other two, upon temperature ramping nucleation first starts at this nanotip. This is supported by the
EBSD mapping in Figure 5d. An $18 \mu \mathrm{m}$-long single crystal grain (in blue) that occupies $\sim 80 \%$ of the entire GeSn microtaper is formed, seeded by the high curvature nanotip on the left. There are grains growing from the other two tips on the right hand side, too, but their sizes much smaller than the dominant single crystal grain. A small amount of sporadic grains mostly form twin boundaries with the dominant single crystal grain. This result proves that the growth of single crystal GeSn on amorphous dielectric layers can indeed be seeded by the high curvature nanotip on an a-GeSn micropattern. Additionally, the microtaper structure in Figures $\mathbf{5 c}, \mathbf{d}$ also facilitates mode conversion and optical coupling with waveguides, as has been applied to waveguide-coupled photodetectors [3, 33].

\section{Sn Nanodot-Induced Composition Enhancement (NICE) in GeSn Crystallization}

In crystallizing blanket a-GeSn thin films, the highest substitutional Sn composition achieved is $\sim 10$ at.\% [10, 32], limited by surface and grain boundary segregation of $\mathrm{Sn}$. 
(a)

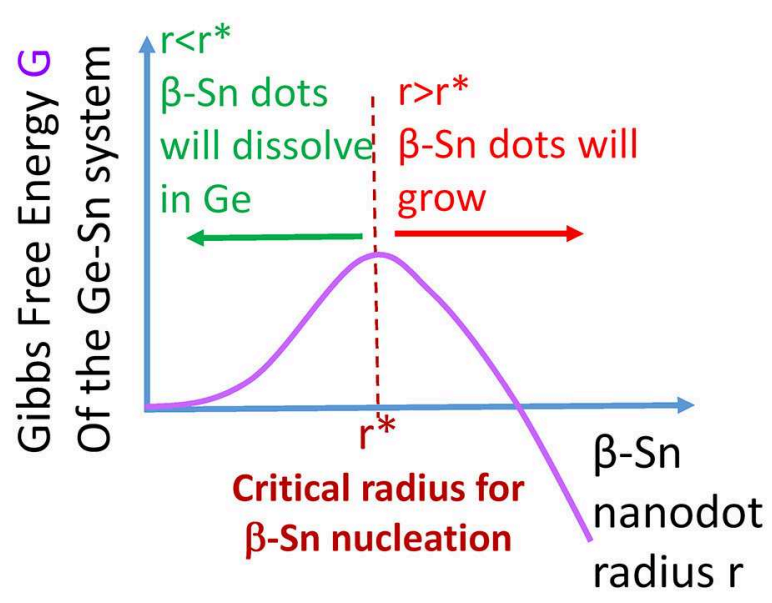

(b)

(1)

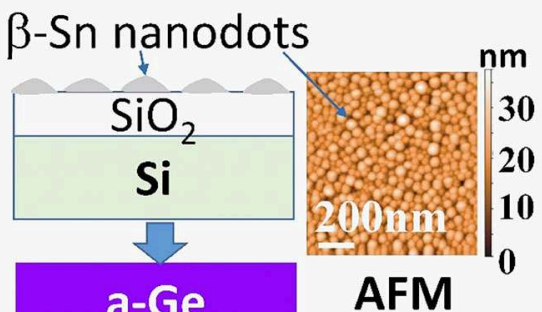

(2)

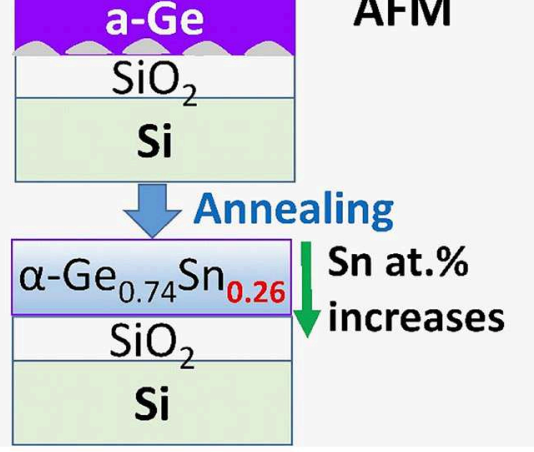

(c)

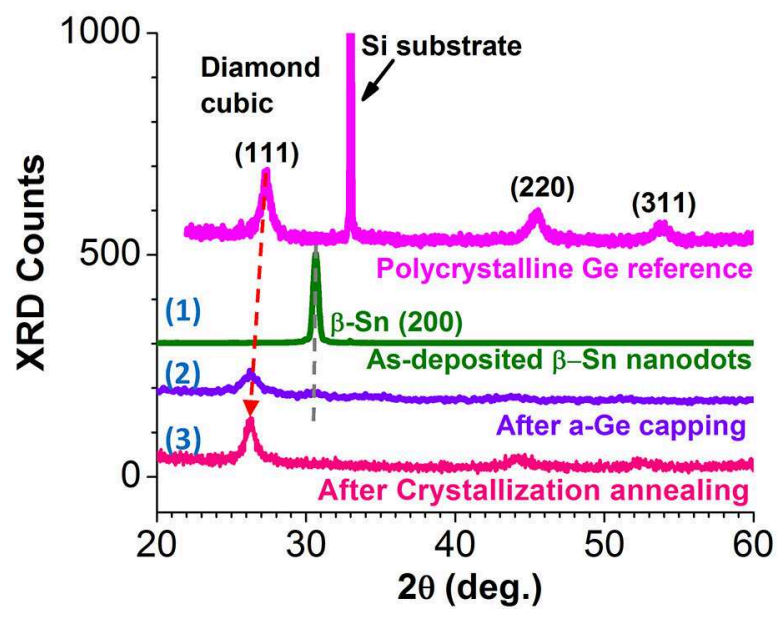

(d)

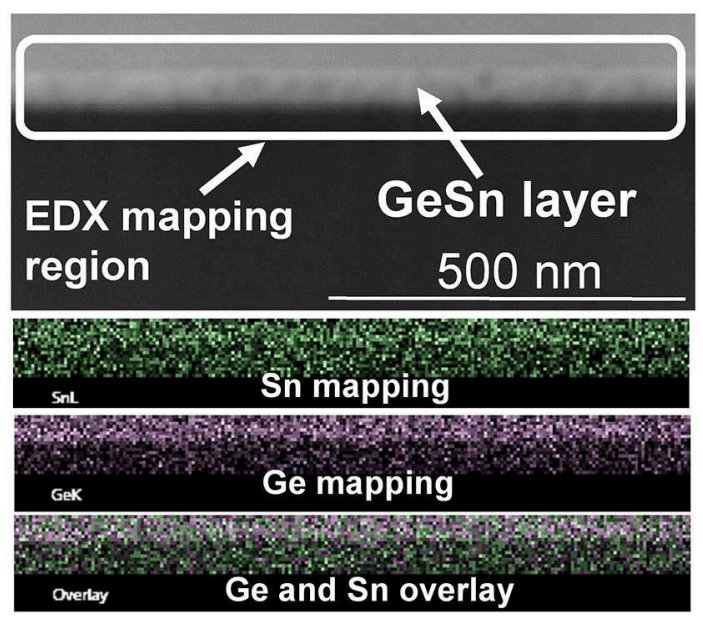

FIGURE 6 | (a) Schematics showing the Gibb free energy of the Ge-Sn system vs. the radius of $\beta$-Sn (semimetal) nanodots. Below the critical size of nucleation $r^{\star}$, the $\beta$-Sn nanodots should dissolve into Ge to minimize the interfacial energy. (b) Schematics showing the NICE fabrication process of crystalline diamond cubic $\alpha$-GeSn with 26 at.\% average Sn. (c) XRD data of a pure Ge reference sample and the Sn or GeSn samples corresponding to steps (1)-(3) in (b). (d) Cross-sectional SEM image (top) and the elemental EDS mapping of Sn, Ge, and Sn-Ge mapping overlay.

RTA can help to suppress Sn segregation and increase the $\mathrm{Sn}$ composition to $\sim 11$ at. $\%$. Using GeSn micropatterns and laserseeded or nanotip-seeded crystallization, the Sn composition has been further increased to $14-15$ at.\% [27, 28] since most of the large-angle grain boundaries, which tend to induce Sn segregation, are eliminated. However, to extend the optical response of $\mathrm{GeSn}$ toward the MIR window at $\lambda=3-5 \mu \mathrm{m}$, an increase of Sn composition beyond 20 at.\% is needed. To address this challenge, we have recently developed NICE growth approach [34, 35]. This method, counter-intuitively, is exactly opposite to the conventional approach of synthesizing diamond cubic GeSn. Since metallic $\beta$-Sn is thermodynamically more stable than diamond cubic $\alpha-\mathrm{Sn}$ at $>13^{\circ} \mathrm{C}$ in bulk materials, conventionally researchers try their best to avoid the nucleation of metallic $\beta$-Sn during GeSn growth. In sharp contrast, this novel NICE approach utilizes a "reverse nucleation" method to dissolve $\beta$-Sn nanodots below the critical size of nucleation into the Ge matrix, thereby achieving an average composition of 26 at. $\% \mathrm{Sn}$ in $\mathrm{Ge}$.

The fundamental principle of the NICE approach is schematically shown in Figure 6a. According to the wellknown nucleation theory, the nucleus of $\beta$-Sn has to reach a critical size, $r^{*}$, in order to implement the phase separation from the diamond cubic matrix of $\mathrm{Ge}$ or $\mathrm{GeSn}$ (labeled as " $\alpha-\mathrm{GeSn}$ " in Figure 6b). Any $\beta$-Sn nucleus smaller than $r^{*}$ would dissolve back into the diamond 
cubic lattice to minimize the Gibbs free energy of the GeSn system. This is because the nucleation of $\beta-S n$ creates new interfaces with the diamond cubic $\alpha-G e S n$ matrix, which increases the interfacial energy that dominates the total Gibbs free energy of nanostructure due to the large surface-to-volume ratio.

Based on this inspiration, if we can first deliberately make $\beta$-Sn nanodots with sizes smaller than $r^{*}$, they will be readily dissolved into $\mathrm{Ge}$ upon $\mathrm{Ge}$ deposition and crystallization annealing, thereby breaking through the existing solubility limit. As schematically shown in Figure 6b, we first deposit a layer of $\beta$-Sn nanodots $(<50 \mathrm{~nm}$ in diameter and $<30 \mathrm{~nm}$ in height, see the inset of Figure $6 \mathbf{b}$ ) on $\mathrm{SiO}_{2} / \mathrm{Si}$, then cap the Sn nanodots with evaporated amorphous Ge (a-Ge). This process artificially creates a huge amount of $\mathrm{Ge} / \mathrm{Sn}$ interfaces. Upon crystallization annealing at $300-500^{\circ} \mathrm{C}$, these nanodots should dissolve into Ge and form crystalline GeSn with high Sn composition in order to eliminate the Ge/Sn interfaces and decrease the total Gibbs free energy (according to Figure 6a). Therefore, the NICE growth approach for high Sn composition is based on dissolving $\beta$-Sn nanodots into the diamond cubic lattice of Ge.

The preliminary demonstration of NICE growth is shown in Figures 6b-d. The inset of Figure $\mathbf{6 b}$ shows an AFM image of nominally $5 \mathrm{~nm}$-thick $\beta$-Sn evaporated onto $\mathrm{SiO}_{2} / \mathrm{Si}$. Sn dewets on $\mathrm{SiO}_{2}$ during the deposition, leading to the selfassembly of nanodots. The average diameter of the Sn dots is $45 \mathrm{~nm}$ and the height is $\sim 30 \mathrm{~nm}$. The XRD data of the asdeposited $\beta$-Sn nanodots are shown in Figure 6c [step (1)], demonstrating a strong (200) preferred orientation. Remarkably, after depositing nominally $20 \mathrm{~nm}$-thick a-Ge on the $\beta$-Sn nanodots, the $\beta$-Sn (200) peak is already almost completely replaced by the (111) peak of nanocrystalline, diamond cubic structured GeSn [step (2)]. Moreover, after crystallization annealing at $450^{\circ} \mathrm{C}$ for $15 \mathrm{~min}$, the $\beta$-Sn (200) peak completely disappears and the $\alpha$-SnGe (111) peak becomes sharper due to improved crystallinity after annealing [step (3)]. Therefore, we can conclude that $\beta$-Sn is completely dissolved into the diamond cubic lattice of GeSn after the crystallization annealing, confirming our hypothesis based on the nucleation theory in Figure 6a. Compared to the pure Ge reference sample, the (111) peak of crystallized GeSn is drastically shifted to lower diffraction angle (indicating a larger lattice constant) due to the significant Sn alloying [Figure 6c, step (3)]. From the peak shift in the XRD data, we estimate an average composition of $26-27$ at.\% Sn in crystallized GeSn. Figure 6d further shows a cross-sectional SEM image and the corresponding EDS mapping of the crystallized GeSn sample grown by NICE approach. The Sn-rich region at the bottom and Ge-rich cap at the top can be clearly observed. An average Sn composition of 26 at.\% Sn is confirmed, and the bottom Sn-rich region shows a Sn composition as high as $\sim 40$ at.\%, indicating that NICE approach has a great potential to achieve even higher Sn composition for covering the entire MIR window of $\lambda=3-5 \mu \mathrm{m}$.

\section{OPTICAL GAIN DYNAMICS OF CRYSTALLIZED DIRECT GAP GeSnOI}

As an approach to evaluate the optoelectronic quality of crystallized GeSnOI discussed in section Substrate-Independent GeSn Crystallization on Amorphous Insulators as well as to elucidate some fundamental questions about the GeSn band structure near the indirect-to-direct gap transition, we performed ultrafast pump-probe optical gain dynamic measurements on the $0.2 \%$ tensile strained $\mathrm{Ge}_{0.913} \mathrm{Sn}_{0.087} \mathrm{OI}$ sample shown in Figure 2. This sample provides a composition close to the indirect-to-direct transition with high crystallinity and large grains on $\mathrm{mm}$-scale so that grain boundaries have negligible effect on the pump-probe measurement. We aim at addressing two fundamental questions:

(1) Is the high lasing threshold observed from optically pumped GeSn lasers mainly due to the intrinsic band structure (i.e., insufficient directness of the bandgap) or is it because of extrinsic defects? Recently, optically pumped GeSn lasers have achieved dramatic progress [17, 19, 20, 22-24], yet the threshold pump power density increases from $\sim 100$ to $1,000 \mathrm{~kW} / \mathrm{cm}^{2}$ as the temperature increases from 77 to $180 \mathrm{~K}$, orders of magnitude higher than bulk homojunction direct gap III-V lasers [36]. Even at $10 \mathrm{~K}$, the threshold pump power density is still $>>10$ $\mathrm{kW} / \mathrm{cm}^{2}$, compared to $<<1 \mathrm{~kW} / \mathrm{cm}^{2}$ from GaAs lasers. The threshold does not show significant decrease even at higher $\mathrm{Sn}$ compositions of 16-18 at.\%, which are supposed to have better direct gap performance, even though the maximal operation lasing temperature does increases. Therefore, it is important to find out the key limiting factors of the lasing threshold.

(2) What is the intrinsic crossover composition for the indirectto-direct bandgap transition in $G e_{1-x} S n_{x}$ ? This issue is still controversial theoretically and experimentally, with the Sn content ranging from 6.5 to 10 at.\% at the transition point from different reports $[9,13,37-39]$. The increase of the integrated photoluminescence (PL) intensity with the decrease of temperatures has been applied as an evidence to prove the direct fundamental bandgap of epitaxial $\mathrm{Ge}_{1-\mathrm{x}} \mathrm{Sn}_{\mathrm{x}}$, in contrast to the behavior of direct gap emission in Ge. However, it has been found recently that there are possible pitfalls associated with this criterion. For example, Pezzoli et al. [40] reported that $\mathrm{Ge}_{0.95} \mathrm{Sn}_{0.05}$ coherently grown on Ge buffer layer (with $-0.8 \%$ compressive strain) also shows a similar PL enhancement at lower temperatures in spite of its indirect gap nature. In fact, it is well-known that even indirect gap semiconductors (such as $\mathrm{Si}$ ) tends to show higher PL intensity at lower temperatures due to less non-radiative recombination. On the other hand, demonstration of lasing can provide evidence for direct gap GeSn at low temperatures, but cannot directly prove that the same composition remains direct gap at room temperature since $\mathrm{GeSn}$ lasers operating at $300 \mathrm{~K}$ are still not available. Thus, direct measurement of the carrier dynamics and optical gain lifetime is required to undoubtedly determine the directness of the fundamental bandgap of $\mathrm{Ge}_{1-\mathrm{x}} \mathrm{Sn}_{\mathrm{x}}$, especially at room temperature. 

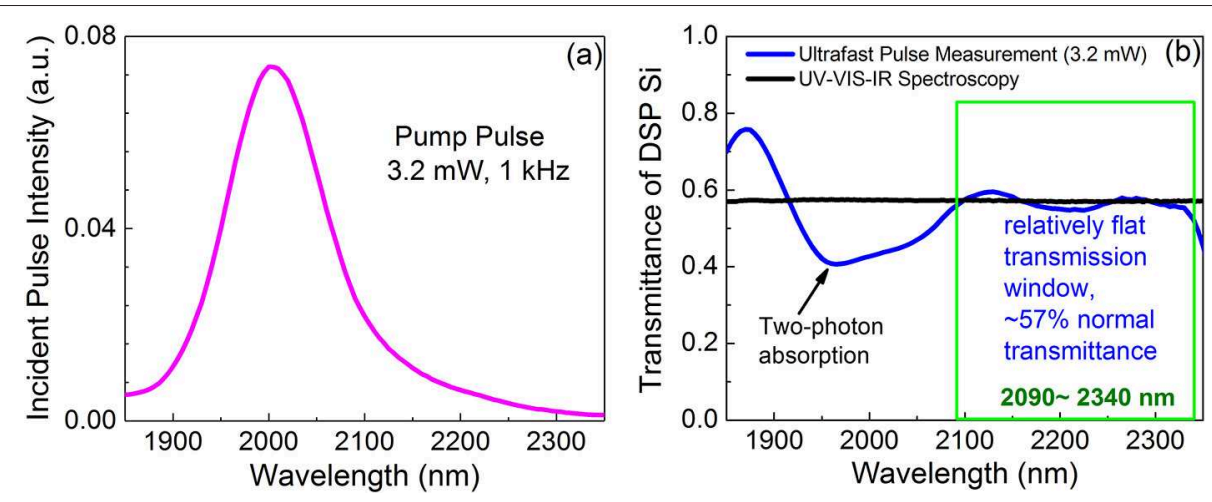

FIGURE 7 | (a) The spectrum of the fs pump pulse with a peak at $\lambda=2,000 \mathrm{~nm}$. The average power is $3.2 \mathrm{~mW}$ and the repetition rate is $1 \mathrm{kHz}$; (b) transmittance spectrum of a double side polished (DSP) Si wafer under the ultrafast pumping condition in (a) (blue line) compared to that measured by UV-VIS-IR spectroscopy (black line). While a dip due to two-photon absorption is observed at $\lambda \sim 1,950-2,050 \mathrm{~nm}$, a relatively flat transmission window with negligible non-linear effect is identified in the range of 2,100-2,340 $\mathrm{nm}$. We will use this range for GeSn optical gain and carrier dynamics studies.

Femtosecond (fs) pump-probe studies provides an ideal approach to address these two fundamental questions. The peak optical gain measured at 0 time delay between the pump and probe pulses provides information about the maximal intrinsic optical gain from the direct band-to-band transitions since the measurement timescale of $<60 \mathrm{fs}$ is faster than any recombination process $[41,42]$. On the other hand, the optical gain lifetime measurement provides affirmative information about the nature of the bandgap (indirect vs. direct) as well as any likely extrinsic factors (e.g., defects) that limits the injected carrier lifetime.

A challenge for ultrafast pump-probe measurement of $\mathrm{GeSn}$, though, is that most of the epitaxial GeSn layers are grown on Ge buffers, which can complicate the pump-probe carrier dynamics analysis due to carrier diffusion between $\mathrm{GeSn}$ and the Ge buffer as well as non-linear effects in Ge such as two-photon absorption (TPA). Direct crystallization of high crystallinity GeSnOI provides a possible approach to circumvent the complication of $\mathrm{Ge}$ buffers. The thin $\mathrm{SiO}_{2}$ layer also prevents photo-generated carrier diffusion from $\mathrm{GeSn}$ into the Si substrate, offering a more accurate evaluation of the injected carrier density. Therefore, ultrafast pump-probe measurements on our GeSnOI samples reveals important information about the optical gain and carrier dynamics inherent to GeSn.

\section{Ultrafast Pump-Probe Measurement Setup}

Our experiments are carried out using a setup similar to what has been described in [29] and [41]. In order to investigate the optical gain spectrum in the $\mathrm{Ge}_{0.913} \mathrm{Sn}_{0.087} \mathrm{OI}$ material, we first measure the power-dependent ultrafast pulse transmittance spectra (at high injection level) and compare it to the linear transmittance spectrum measured by a Jasco V-570 UV-VISIR spectrometer with a Jasco ISN-470 integrating sphere (at negligible injection level). For the ultrafast measurements, the incident light consist of broadband, $<60$ fs pulses covering the wavelength range of $1,850-2,400 \mathrm{~nm}$. These are the idler pulses from an optical parametric amplifier (OPA, Spectra-Physics OPA-800C) pumped by high-energy pulses at $\lambda=800 \mathrm{~nm}$ seeded by a Spectra-Physics Tsunami Ti:Sapphire laser and amplified by Spectra-Physics Spitfire-Pro amplifier. In power-dependent ultrafast transmittance spectra measurements, the focal spot is in Gaussian profile with a beam waist of $90 \mu \mathrm{m}$, as measured by razor-blade method. In pump-probe measurements, the pump beam spot waist is increased to $200 \mu \mathrm{m}$ in order to make sure that the probe spot overlaps with it in the center. Since the focal spot size is much smaller than the grain size of $\sim 1 \mathrm{~mm}$, we adjust the beam position so that it is focused within a single grain during the pump-probe measurement. The transmitted light was then detected by a thermoelectric (TE)-cooled (at $-65^{\circ} \mathrm{C}$ ) $\mathrm{PbS}$ detector with responsivity covering the wavelength range of 1$3 \mu \mathrm{m}$. The detector output was amplified by a lock-in amplifier triggered by the pulse repetition frequency of $1 \mathrm{kHz}$.

Figure $7 \mathrm{a}$ shows the spectrum of the $<60 \mathrm{fs}$ incident pulse used in this study. The higher energy photons near the peak wavelength of the pulse at $\lambda \sim 2,000 \mathrm{~nm}$ inject a large amount of carriers into the GeSn thin films, thereby inducing population inversion, optical bleaching and optical gain for the lower energy photons in the same pulse near the direct band gap of $\mathrm{Ge}_{0.913} \mathrm{Sn}_{0.087}$ at $\lambda=2,200-2,300 \mathrm{~nm}$. This measurement is a good approach to probe the maximal transient gain with minimal time delay between the excitation of the higher energy photon and the transmission of the lower energy photons since they arrive at the sample within the duration of the $<60 \mathrm{fs}$ pulse. The peak wavelength of the incident pulse located at $2,000 \mathrm{~nm}$ is far away from the 2,200 to $2,300 \mathrm{~nm}$ wavelength range for transmittance spectra measurement, thereby avoiding any artifact in the gain spectrum due to its overlap with the peak of the pump pulse.

To make sure that the incident pulse does not introduce any complicated non-linear effect in the double-side polished (DSP) Si substrate itself in the wavelength range of interest $(\lambda=2,200-$ $2,300 \mathrm{~nm}$ ), we first measured the fs pump transmittance spectrum of the Si DSP wafer as a reference, as shown in Figure $7 \mathbf{b}$. Corresponding to the peak of the pumping wavelength, we see a dip at 1,950-2,050 nm due to strong TPA in DSP Si. On the other hand, the TPA coefficient $\beta$ tends to be zero 

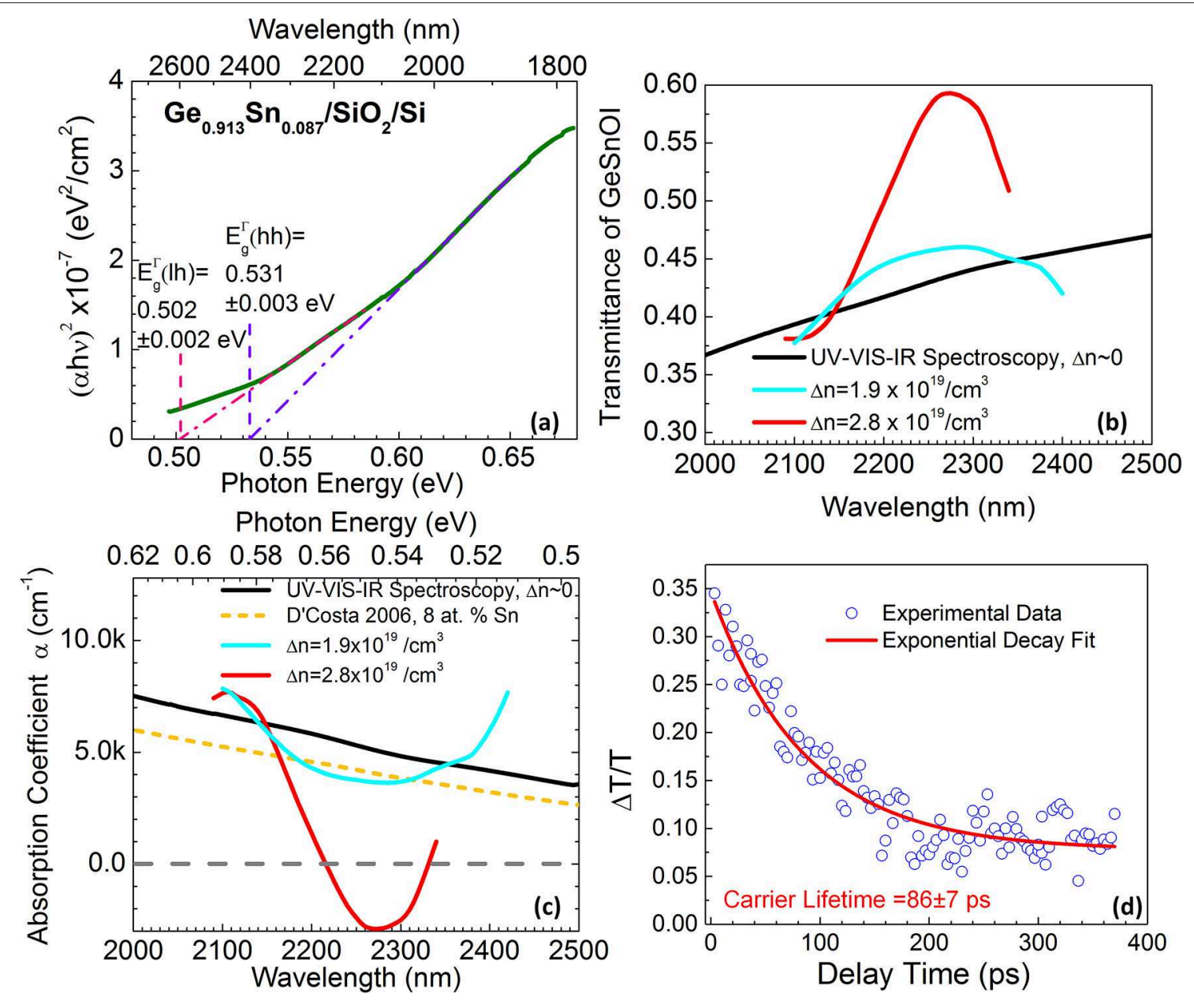

FIGURE 8 | (a) Linear fit to $(\alpha \mathrm{h} v)^{2}$ vs. hv indicates that crystalized $\mathrm{Ge}_{0.913} \mathrm{Sn}_{0.087}$ has a direct band gap of $\mathrm{E}_{\mathrm{g}}^{\Gamma}$ (Ih) $=0.502 \pm 0.002 \mathrm{eV}$ and $\mathrm{E}_{\mathrm{g}}^{\Gamma}$ (Ih) $=0.531 \pm$ $0.003 \mathrm{eV}$. Here $\alpha$ is absorption coefficient, and hv is photon energy. (b) Transmittance spectrum of the GeSnOI sample measured by UV-VIS-IR spectroscopy (black line) compared to the ultrafast pulse transmittance spectra of the same sample under injected carrier density of $\Delta n=1.9 \times 10^{19} / \mathrm{cm}^{3}$ (cyan line) and $\Delta n=2.8 \times$ $10^{19} / \mathrm{cm}^{3}$ (red line). (c) Comparison of derived absorption coefficients of crystalized $\mathrm{Ge}_{0.913} \mathrm{Sn}_{0.087} \mathrm{OI}$ with UV-VIS-IR spectroscopy (no pumping, black line), and under injected carrier densities $\Delta n=1.9 \times 10^{19} / \mathrm{cm}^{3}$ (cyan line) and $\Delta n=2.8 \times 10^{19} / \mathrm{cm}^{3}$ (red line). Negative absorption coefficient (below the dotted gray line) indicates a net optical gain. As a comparison, the linear absorption coefficient of epitaxial GeSn with 8 at.\% Sn on Si reported by D'Costa et al. [37] is also shown (short dashed orange line). (d) Results of gain lifetime measurement plotting pump-induced change in transmittance $(\Delta T / T)$ vs. the delay time $(\Delta t)$. The pump pulse is peaked at $2,000 \mathrm{~nm}$, and the probe wavelength is $2,300 \mathrm{~nm}$. The injected carrier density is estimated to be $\Delta n=4.8 \times 10^{19} \mathrm{~cm}^{-3}$.

for wavelengths larger than 2,200 $\mathrm{nm}$, corresponding to photon energies less than half of the Si band gap [43]. Indeed, the nearly flat transmittance spectrum in the wavelength range of 2,1002,340 $\mathrm{nm}$ shows no non-linear effect from the DSP Si substrate, consistent with the linear transmittance spectrum measured by UV-VIS-IR spectroscopy. Therefore, this measurement confirms that the ultrafast transmittance data between 2,090 and 2,340 nm will be free of non-linear effects from the substrate, and that any optical gain in this wavelength range is attributed to the GeSn layer on top of Si.

\section{Ultrafast Optical Gain and Carrier Dynamics Measurement of $\mathrm{Ge}_{0.913} \mathrm{Sn}_{0.087} \mathrm{OI}$} Before analyzing the absorption spectra under ultrafast pulse pumping, we first derived the absorption coefficient spectrum of the $\mathrm{Ge}_{0.913} \mathrm{Sn}_{0.087} \mathrm{OI}$ sample from the transmittance spectrum measured by UV-VIS-IR spectrometer utilizing iterative selfconsistent solution method based on transfer matrix method and Kramer-Kronig relation [41]. This analysis gives the baseline absorption of $\mathrm{Ge}_{0.913} \mathrm{Sn}_{0.087}$ without optical pumping, i.e., at an injected carrier density of $\Delta n \sim 0$. The result is shown in Figure 8a. For direct gap optical absorption, it is well-known that

$$
(\alpha h v)^{2} \propto\left(h v-E_{g}\right)
$$

Here $\alpha$ is the absorption coefficient, $h v$ is the photon energy, and $E_{g}$ is the direct bandgap. Since the $0.2 \%$ biaxial tensile strain splits the light hole $(l h)$ and heavy hole $(h h)$ bands at the center of the Brillouin zone $(k=0)$, the crystalized $\mathrm{Ge}_{0.913} \mathrm{Sn}_{0.087}$ shows 
a direct band gap of $E g^{\Gamma}(l h)=0.502 \mathrm{eV}$ and $E g^{\Gamma}(h h)=0.531 \mathrm{eV}$. The obtained direct gaps are close to the expected value of $0.52 \mathrm{eV}$ for $8.7 \% \mathrm{Sn}$ based on the report of [37]. Figure 8c further shows that the derive absorption spectrum of $\mathrm{Ge}_{0.913} \mathrm{Sn}_{0.087}$ at $\lambda=2,000-2,500 \mathrm{~nm}$ is also similar to the data for epitaxial $\mathrm{Ge}_{0.92} \mathrm{Sn}_{0.08}$ samples reported by [37]. Below its direct band gap of $0.54 \mathrm{eV}$, the absorption coefficient from the epitaxial $\mathrm{Ge}_{0.92} \mathrm{Sn}_{0.08}$ layer is still high, e.g., $2,500 \mathrm{~cm}^{-1}$ at $0.50 \mathrm{eV}$ [37], which is believed to be Urbach tail absorption. Our crystalized $\mathrm{Ge}_{0.913} \mathrm{Sn}_{0.087}$ film has an additional $1,000 \mathrm{~cm}^{-1}$ absorption at $h v$ $=0.50 \mathrm{eV}$ compared to its epitaxial counterpart in [37], partially due to higher Sn composition ( $\sim 9$ vs. 8 at.\%) and partially due to Urbach tail below the direct gap that is clearly shown in Figure 8a. The Urbach tail absorption loss, probably originated from the nanoscale disorder of Sn distribution in the material, could be a limiting factor for the demonstration of low-threshold GeSn lasers.

Next, we measured the ultrafast pulse transmittance spectra of the GeSnOI sample at different incident power/injection levels to reveal the intrinsic gain from the direct gap transition of $\mathrm{GeSn}$ using the pulse spectrum shown in Figure 7a. This captures the maximal optical gain within the $60 \mathrm{fs}$ pulse width of the injection, practically before any carrier recombination could occur [41, 42], thereby revealing the nature of the intrinsic band structure. Figure $\mathbf{8 b}$ shows that, in the wavelength regime of 2,100-2,340 $\mathrm{nm}$ free of non-linear effects of the substrate (see Figure $7 \mathbf{b}$ ), we clearly observe strongly enhanced transmittance peak (red curve) at an injected carrier density of $\Delta n \sim 2.8 \times$ $10^{19} \mathrm{~cm}^{-3}$ from ultrafast pulse pumping compared to the regular transmittance (i.e., negligible injection level $\Delta n \sim 0$ ) measured by UV-VIS-IR spectroscopy. The injected carrier density is estimated based on the input optical pulse power, the measured beam profile, and the measured thickness and optical absorption of GeSn at this wavelength. The transmittance is enhanced by $\sim 37 \%$ at the peak wavelength of $2,270 \mathrm{~nm}$ at an injection level of $\Delta n \sim 2.8 \times 10^{19} \mathrm{~cm}^{-3}$. The peak transmittance reaches 0.593 at $2,270 \mathrm{~nm}$, even higher than that of the DSP Si substrate (0.572). As will be shown later in our quantitative analyses, this is due to the large optical gain in the GeSn thin film under high injection level. With a similar method, we also studied the case of lower injected carrier density. Under $\Delta n=1.9 \times 10^{19} / \mathrm{cm}^{3}$, the transmittance becomes lower compared to the case of $\Delta n=2.8 \times$ $10^{19} / \mathrm{cm}^{3}$, as expected. The peak transmittance occurs at a longer wavelength of $\lambda=2,290 \mathrm{~nm}$ compared to $\lambda=2,270 \mathrm{~nm}$ for $\Delta n$ $=2.8 \times 10^{19} / \mathrm{cm}^{3}$. Clearly, the transmittance peak blueshifts and the magnitude increases with the pump power. This is consistent with the band filling upon population inversion, again proving that the enhanced transmittance is due to the optical bleaching or gain in crystalized $\mathrm{Ge}_{0.913} \mathrm{Sn}_{0.087}$ films.

Further utilizing iterative self-consistent solution of the absorption spectra considering Kramer-Kronig relation and transfer matrix method [41], we are able to obtain the absorption/gain spectra at different injection levels, as shown in Figure 8c. Under $\Delta n=2.8 \times 10^{19} / \mathrm{cm}^{3}$ injection, a net gain (i.e., negative absorption) is observed in the wavelength range of $2,220-2,330 \mathrm{~nm}$ at room temperature from the crystallized $\mathrm{Ge}_{0.913} \mathrm{Sn}_{0.087} \mathrm{OI}$. The peak gain coefficient is $\sim 2,900 \mathrm{~cm}^{-1}$ at
2,270 nm, comparable to III-V semiconductors and promising for room-temperature lasing. On the other hand, under a lower injection level of $\Delta n=1.9 \times 10^{19} / \mathrm{cm}^{3}$, only optical bleaching is observed with the absorption coefficient decreasing from 4,800 to $3,680 \mathrm{~cm}^{-1}$ at $2,300 \mathrm{~nm}$. This comparison indicates that $\mathrm{Ge}_{0.913} \mathrm{Sn}_{0.087}$ needs $\sim 10 \mathrm{x}$ larger injected carrier density for optical transparency than III-V semiconductors, i.e., $\Delta n_{0} \sim 2$ $\times 10^{19} / \mathrm{cm}^{3}$ in GeSn vs. $\Delta n_{0} \sim 2 \times 10^{18} / \mathrm{cm}^{3}$ in GaAs, possibly due to a larger optical loss (e.g., from free carriers and intervalence band transitions). The high injection level needed for transparency could be one of the limiting factors of the lasing threshold.

To further study the carrier dynamics in GeSnOI, the ultrafast pulse with the peak positioned at 2,000 nm is used as the pump, and the probe wavelength is set at $2,300 \mathrm{~nm}$. The experimental setup is similar to [29], except that the probe is shifted to longer wavelengths closer to the direct gap of $\mathrm{Ge}_{0.913} \mathrm{Sn}_{0.087}$ shown in Figure 8a, while the pump is shifted to shorter wavelengths to better distinguish from the probe. The probe pulse is incident vertically on the sample, while the pump pulse is incident at 60 degree angle. The beam profiles of the pump and probe spots on the surface of the sample are measured by razor-blade method and carefully overlapped, with the spot size of the pump pulse $(200 \mu \mathrm{m})$ being $\sim 2 \mathrm{x}$ that of the probe spot $(90 \mu \mathrm{m})$. An aperture is placed immediately at the backside of the sample to block any astray light from the pump, thereby gathering the probe signal only. A neutral density (ND) filter is put in the probe path to achieve a pump-to-probe intensity ratio $>8: 1$ and also to ensure no non-linear effect caused by the probe itself. The pump-induced change in the transmittance of the probe, $\Delta T$, is normalized to the transmittance without pumping $(T) . \Delta T / T$ is measured as a function of delay time $\Delta t$ between the probe pulse and the pump pulse to evaluate the carrier dynamics and optical gain lifetime in the sample by varying the optical path length of the probe with a translation stage in the probe path.

At an injection level of $\Delta n=4.8 \times 10^{19} \mathrm{~cm}^{-3}$ and a wavelength of $\lambda=2,300 \mathrm{~nm}$, we observe $\Delta T / T$ as a function of the delay time $\Delta t$ between the probe and pump, as shown in Figure 8d. $\Delta T / T$ is about 0.35 at $\Delta t \sim 0$, corresponding to a net gain of $3,500 \mathrm{~cm}^{-1}$ at $2,300 \mathrm{~nm}$, and it decays exponentially with delay time $\Delta t$. Therefore, Figure $\mathbf{8 d}$ is a direct measurement of the gain lifetime. In the case of indirect gap intrinsic Ge, the phonon-assisted carrier scattering from the direct $\Gamma$ valley to the indirect $\mathrm{L}$ valleys limits the gain lifetime to as short as $\sim 200$ fs [44-46]. In our case of $\mathrm{Ge}_{0.913} \mathrm{Sn}_{0.087} \mathrm{OI}$, on the other hand, Figure 8d clearly shows a much longer gain lifetime of $86 \pm 7 \mathrm{ps}$, nearly 3 orders of magnitude longer than indirect gap Ge. This result clearly confirms that $0.2 \%$ tensile strained $\mathrm{Ge}_{0.913} \mathrm{Sn}_{0.087}$ is indeed a direct gap semiconductor at room temperature. Measurements at other pumping wavelengths between 2,200 and $2,350 \mathrm{~nm}$ also indicate a gain lifetime on the order of 70-100 ps. Note that this gain lifetime is on the same order as the carrier lifetime of epitaxial GeSn lasers, i.e., 350 ps from [17]. This result demonstrates that the optoelectronic quality of crystallized GeSn is approaching their epitaxial counterparts. For laser applications, ideally the gain lifetime should reach several ns to achieve a reasonable threshold. Therefore, the short gain lifetime on the 

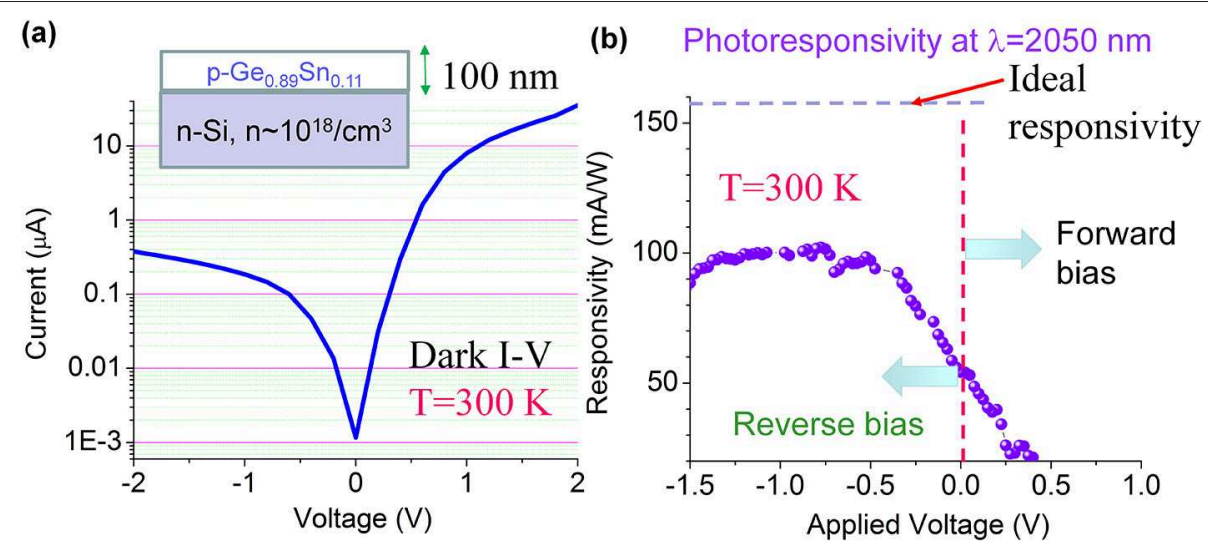

FIGURE 9 | (a) I-V characteristics of the crystallized p-Ge $0.89 \mathrm{Sn}_{0.11} / \mathrm{n}$-Si photodiode. (b) Responsivity vs. applied voltage at an excitation wavelength of $\lambda=2,050 \mathrm{~nm}$.

order of $0.1 \mathrm{~ns}$ in GeSn is another key limiting factor for lasing at room temperature. There are two possible reasons for the short gain lifetime in GeSn: (1) Auger recombination at high injection levels $\left(\Delta n>10^{19} \mathrm{~cm}^{-3}\right)$ in small band gap GeSn ( $E_{g} \sim 0.5 \mathrm{eV}$; see Figure 8a); (2) non-radiative recombination due to extrinsic defects in GeSn. Note that there could be some convolution between these two factors since defects could also assist Auger process.

Differential gain is another main parameter responsible for the quantum efficiency. It is the derivative of material gain vs. the injected carrier density evaluated near the lasing threshold. From Figures $\mathbf{8 c}, \mathbf{d}$, the maximal gain at $\Delta t=0$ is enhanced from 2,500 to $3,600 \mathrm{~cm}^{-1}$ at $2,300 \mathrm{~nm}$ when the injected carrier density is increases from $2.8 \times 10^{19}$ to $4.8 \times 10^{19} \mathrm{~cm}^{-3}$. This gives a differential gain of $\sim 5.5 \times 10^{-17} \mathrm{~cm}^{2}$, one order of magnitude lower than bulk GaAs lasers.

In summary, our ultrafast pulse transmittance and pumpprobe spectroscopy studies confirm that $0.2 \%$ tensile strained GeSn with 9 at.\% Sn is already a direct gap semiconductor at room temperature. The direct band gap transition in GeSn leads to a large intrinsic gain of $\sim 3,000 \mathrm{~cm}^{-1}$, comparable to III$\mathrm{V}$ semiconductors and promising for room-temperature lasing. However, a high transparency threshold of $\Delta n_{0}>2 \times 10^{19} \mathrm{~cm}^{-3}$, a short gain lifetime on the order of $100 \mathrm{ps}$, and a relatively small differential gain of $\mathrm{Ge}_{0.91} \mathrm{Sn}_{0.09}$ material pose challenges for the development of low-threshold room temperature GeSn lasers. The impact of these three major factors can be manifested by estimating the threshold current density for an ideal laser device assuming $100 \%$ optical confinement in the GeSn gain medium [47]:

$$
J_{t h}=\frac{\Delta n_{0} q d}{\tau_{r}}+\frac{\alpha_{i}}{\Gamma}+\frac{1}{2 \Gamma A L} \ln \left(\frac{1}{R_{1} R_{2}}\right)
$$

Here $\tau_{r}$ is the carrier lifetime, $d$ is the GeSn layer thickness, $\Delta n_{0}$ is the injected carrier density for transparency, $q$ is the electron charge, $\alpha_{i}$ is the internal loss, $\Gamma$ is the confinement factor, $L$ is the cavity length, $g$ is the differential gain at the transparency, $A=\frac{g \tau_{r}}{q d}$, and $R_{1}$ and $R_{2}$ are the front and back mirror reflectivities.
Based on the parameters obtained in our pump-probe studies and assuming ideal optical confinement in $\mathrm{GeSn}$, we use the following values at $\lambda=2,300 \mathrm{~nm}: \Delta n_{0}=2 \times 10^{19} \mathrm{~cm}^{-3}, \tau_{r}=100 \mathrm{ps}$, $g=5.5 \times 10^{-17} \mathrm{~cm}^{2}, q=1.6 \times 10^{-19} \mathrm{C}, d=300 \mathrm{~nm}$, $\Gamma=1, \alpha_{i}=1000 \mathrm{~cm}^{-1}$ considering the Urbach tail absorption and the free carrier absorption, $L=200 \mu \mathrm{m}, R_{1}=90 \%$, and $R_{2}=100 \%$. The estimated threshold current density of GeSn lasers operating at room temperature would be $\sim 2,000 \mathrm{kA} / \mathrm{cm}^{2}$, equivalent to a threshold on the order of $\mathrm{MW} / \mathrm{cm}^{2}$ under optical pumping. This estimate of lasing threshold is consistent with the experimental result demonstrated recently at $270 \mathrm{~K}$ from a higher Sn composition [24]. Therefore, our ultrafast pump-probe analysis does reveal the major factors limiting the achievement of low threshold GeSn lasers at room temperature. Based on this analysis, we suggest that further reducing the defects in GeSn could get rid of the extrinsic absorption loss from the Urbach tail (which could still exist in epitaxial GeSn [37]) and increase the gain lifetime, thereby reducing the lasing threshold and truly employing the large intrinsic optical gain of direct gap GeSn. The relation between Auger recombination and extrinsic defect and intrinsic band structures (e.g., the transition between the first and second conduction valleys mentioned in section Blanket GeSn Thin Film Crystallization) needs further evaluation. Upconversion spectroscopy could be applied to investigate the latter.

\section{PROTOTYPE MIR PHOTODETECTORS BASED ON CRYSTALLIZED GeSn}

Based on the high crystallinity GeSnOI materials discussed in section Substrate-Independent GeSn Crystallization on Amorphous Insulators and tested optically in section Optical Gain Dynamics of Crystallized Direct Gap GeSnOI, we demonstrate a $\mathrm{p}-\mathrm{Ge}_{0.89} \mathrm{Sn}_{0.11} / \mathrm{n}$-Si $\mathrm{p}$-n photodiode working at $\lambda=2,050 \mathrm{~nm}$ in Figure 9. The sample is prepared by RTA crystallization of $100 \mathrm{~nm}$-thick a-GeSn on n-Si substrate at $450^{\circ} \mathrm{C}$, leading to a $\mathrm{Sn}$ incorporation of 11 at.\%. To maintain the beneficial features of GeSn crystallized on $\mathrm{SiO}_{2}$ [e.g., (111) preferred orientation to enhance the beneficial effect of tensile 
TABLE 1 | Summary of the recent demonstration of GeSn detectors compared to free-space Ge p-i-n photodiodes.

\begin{tabular}{|c|c|c|c|c|c|}
\hline References & Structure & $x_{\text {Sn }}(\%)$ & $\begin{array}{l}\text { Dark current } A / \mathrm{cm}^{2} \\
\text { at }-1 \mathrm{~V}\end{array}$ & Responsivity A/W & Speed \\
\hline Werner et al. [48] & $p-i-n$ & 0.5 (MBE) & 10 & $\begin{array}{l}0.102 @ 0 V, 1,550 \mathrm{~nm} \\
0.018 @ 0 V, 1,640 \mathrm{~nm}\end{array}$ & - \\
\hline Roucka et al. [49] & $p-i-n$ & 2.0 (UHCVD) & $\sim 10$ & $\begin{array}{l}0.113 @ 0 V, 1,550 \mathrm{~nm} \\
0.086 @ 0 \mathrm{~V}, 1,640 \mathrm{~nm}\end{array}$ & - \\
\hline Su et al. [50] & $p-i-n$ & 3 (MBE) & 2.36 & $\begin{array}{l}0.23 @-1 \mathrm{~V}, 1,540 \mathrm{~nm} \\
0.12 @-1 \mathrm{~V}, 1,640 \mathrm{~nm}\end{array}$ & - \\
\hline Oehme et al. [51] & $p-i-n$ & 4 (MBE) & 100 & $\begin{array}{l}0.181 @-0.1 \mathrm{~V}, 1,550 \mathrm{~nm} \\
0.171 @-0.1 \mathrm{~V}, 1,600 \mathrm{~nm}\end{array}$ & - \\
\hline Oehme et al. [52] & $p-i-n$ & 4.2 (MBE) & 0.9 & $0.218 @-1 \mathrm{~V}, 1,550 \mathrm{~nm}$ & $\begin{array}{l}40 \mathrm{GHz} @ \\
-5 \mathrm{~V} \\
1,550 \mathrm{~nm}\end{array}$ \\
\hline Pham et al. [53] & $p-i-n$ & $\begin{array}{l}6.4 \text { (CVD) } \\
9.2 \text { (CVD) }\end{array}$ & $\begin{array}{c}5.74 \\
18\end{array}$ & $\begin{array}{l}0.3 @ 0 \mathrm{~V}, 1,550 \mathrm{~nm} \\
0.19 @ 0 \mathrm{~V}, 1,550 \mathrm{~nm} \\
\text { Cutoff } 2.6 \mu \mathrm{m}\end{array}$ & \\
\hline Gassenq et al. [54] & MSM & $\begin{array}{l}\mathrm{Ge}_{0.91} \mathrm{Sn}_{0.09} / \mathrm{Ge} \\
\text { quantum well (CVD) }\end{array}$ & - & $0.1 @-5 \mathrm{~V}, 2.2 \mu \mathrm{m}$ & $2 \mathrm{MHz}$ \\
\hline Dong et al. [55] & $p-i-n$ & $\begin{array}{l}\mathrm{Ge}_{0.9} \mathrm{Sn}_{0.1} / \mathrm{Ge} \mathrm{MQW} \\
\text { (CVD) }\end{array}$ & 0.031 & $\begin{array}{l}0.216 @-1 \mathrm{~V}, 1,530 \mathrm{~nm} \\
0.023 @-1 \mathrm{~V}, 2 \mu \mathrm{m}\end{array}$ & $1.2 \mathrm{GHz}$ \\
\hline Peng et al. [56] & $\begin{array}{l}\text { waveguide } \\
\text { coupled p-i-n }\end{array}$ & 1.28 (CVD) & 6.75 & $\begin{array}{l}0.27 @ 0 V, 1,550 \mathrm{~nm} \\
0.124 @ 0 \mathrm{~V}, 1,600 \mathrm{~nm}\end{array}$ & - \\
\hline This work & $\begin{array}{l}\mathrm{p}- \\
\mathrm{Ge}_{0.89} \mathrm{Sn}_{0.11} / \mathrm{n}- \\
\mathrm{Si}\end{array}$ & 11 & 0.001 & $0.1 @-1$ V, 2,050nm & - \\
\hline Liu et al. [57] & p-i-n Ge/Si & $\begin{array}{l}0 \text { (UHCVD) } 0.2 \% \\
\text { tensile strained Ge }\end{array}$ & 0.01 & $\begin{array}{l}0.56 @ 0 V, 1,550 \mathrm{~nm} \\
0.11 @ 0 \mathrm{~V}, 1,605 \mathrm{~nm}\end{array}$ & $\begin{array}{l}8.5 \mathrm{GHz} @ \\
-1 \mathrm{~V}\end{array}$ \\
\hline
\end{tabular}

strain [21]], we did not deliberately remove the native oxide on fresh $\mathrm{Si}$ wafers before the a-GeSn deposition. The active area of the device is $200 \times 200 \mu \mathrm{m}^{2}$. The I-V characteristics of the device is shown in Figure 9a, with a clear rectifying behavior and a dark current as low as $200 \mathrm{nA}$ at $-1 \mathrm{~V}$ reserve bias. This corresponds to a dark current density of $<1 \mathrm{~mA} / \mathrm{cm}^{2}$ at $1-2 \mathrm{~V}$ reverse bias, approaching the level of epi $\mathrm{Ge} / \mathrm{Si}$ photodiodes and better than most of the existing epitaxial GeSn photodetectors on $\mathrm{Si}$ (see Table 1). Figure $\mathbf{9 b}$ shows the responsivity of the device vs. applied voltage under the optical excitation of an LED emitting at $\lambda=2,050 \mathrm{~nm}$. Clear photovoltaic behavior is observed, and full responsivity is observed at a low reverse bias of $-1 \mathrm{~V}$. Due to the limited thickness of the GeSn MIR absorber layer $(\sim 100 \mathrm{~nm}$ thick), the absorption at $\lambda=2,050 \mathrm{~nm}$ was measured to be $\sim 9 \%$ by UV-VIS-IR spectrometer. Therefore, in the ideal case the responsivity would be $150 \mathrm{~mA} / \mathrm{W}$, compared to the experimental result of $100 \mathrm{~mA} / \mathrm{W}$ (Figure 9b). This result indicates a carrier collection efficiency of $\sim 67 \%$. Note that the responsivity at $2,050 \mathrm{~nm}$ is already on the same order as some commercial $\mathrm{PbS}$ photodetectors. According to [58], the peak photoresponse of a $\mathrm{PbS}$ photoconductor at 2,200 nm wavelength under $15 \mathrm{~V}$ bias is $5 \times 10^{4} \mathrm{~V} / \mathrm{W}$, with a load resistance $=$ dark resistance $=0.25-2.5 \mathrm{M} \Omega$. This translates to a responsivity of 20$200 \mathrm{~mA} / \mathrm{W}$ (i.e., $5 \times 10^{4} \mathrm{~V} / \mathrm{W}$ divided by $0.25-2.5 \mathrm{M} \Omega$ ). The fact that we already achieved $100 \mathrm{~mA} / \mathrm{W}$ responsivity at a much lower bias of $1 \mathrm{~V}$ is clearly very competitive to the existing technology and high promising for future improvement. We will further investigate $\mathrm{GeSn}$ crystallization on n-type conductive oxides on flexible substrates in our future work to check if similar device performance can be maintained for flexible MIR photonics.

\section{CONCLUSIONS}

In conclusion, we have demonstrated low-temperature crystallization of direct bandgap, high crystallinity $\mathrm{Ge}_{1-\mathrm{x}} \mathrm{Sn}_{\mathrm{X}}$ $(0.08<\mathrm{x}<0.26)$ on amorphous dielectrics insulators (GeSnOI) toward 3D MIR photonic integration as well as flexible MIR photonics. Utilizing EEC, an extraordinarily large average grain size of $\sim 100 \mu \mathrm{m}$ has been achieved in blanket GeSn films crystallized on $\mathrm{SiO}_{2}$ layers, flexible glass, fused silica, and polyimide substrates alike. Furthermore, using Sn nanodot enhanced composition enhancement, we have achieved an average GeSn composition as high as 26 at.\% to further extend the optical response of GeSn toward $\lambda=3-5 \mu \mathrm{m}$. The achieved Sn composition of 8-26 at.\% far exceeds that of the equilibrium solubility limit of $<1$ at.\%, even though the crystallization temperature of $350-450^{\circ} \mathrm{C}$ far exceeds the typical epitaxial growth temperature of GeSn to prevent Sn segregation. This result indicates that crystallization from a-GeSn may offer better metastability compared to direct epitaxial growth of $\mathrm{GeSn}$. Attesting to the high crystallinity, a peak optical gain of 2,900 $\mathrm{cm}^{-1}$ with a lifetime on the order of $0.1 \mathrm{~ns}$ is achieved at $\lambda$ $=2,200-2,350 \mathrm{~nm}$ at $300 \mathrm{~K}$. The intrinsic gain coefficient is comparable to III-V semiconductors. The gain lifetime is on the same order as epitaxial GeSn, and it is $>100 x$ longer than the direct gap transition in Ge, confirming the indirect-to-direct 
band gap transition in GeSn at $\sim 9$ at. Sn composition. The study also suggests that further reducing the defects in GeSn could greatly reduce the Urbach tail absorption losses and increase the gain lifetime toward practical laser devices. Moreover, a prototype $\mathrm{p}-\mathrm{GeSn} / \mathrm{n}$-Si photodiode from a-GeSn crystallization achieves $100 \mathrm{~mA} / \mathrm{W}$ responsivity at $\lambda=2,050 \mathrm{~nm}$ and $\mathrm{T}=$ $300 \mathrm{~K}$, approaching the level of some commercial PbS detectors. The device also demonstrates photovoltaic behavior and a low dark current density of $1 \mathrm{~mA} / \mathrm{cm}^{2}$ at $-1 \mathrm{~V}$ reverse bias, comparable to epitaxial $\mathrm{Ge} / \mathrm{Si}$ photodiodes. These results indicate that crystallization of GeSnOI offers a promising solution for active devices toward 3D MIR photonic integration and flexible MIR photonics.

\section{DATA AVAILABILITY STATEMENT}

The datasets generated for this study are available on request to the corresponding author.

\section{AUTHOR CONTRIBUTIONS}

XW performed optical and ultrafast pump-probe characterization of the GeSn samples. She also contributed significantly to the write-up of this paper. AC and $\mathrm{HL}$

\section{REFERENCES}

1. Soref RA. The past, present, and future of silicon photonics. IEEE J Sel Topics Quantum Electron. (2006) 12:1678-87. doi: 10.1109/JSTQE.2006.8 83151

2. Subbaraman H, Xu X, Hosseini A, Zhang X, Zhang Y, Kwong D, et al. Recent advances in silicon-based passive and active optical interconnects. Opt Express. (2015) 23:2487-511. doi: 10.1364/OE.23.002487

3. Liu J. Monolithically integrated Ge-on-Si active photonics. Photonics. (2014) 1:162-97. doi: 10.3390/photonics1030162

4. Wada K, Kimerling LC. Photonics and Electronics With Germanium. Weinheim: Wiley-VCH (2015). doi: 10.1002/9783527650200

5. Wang X, Liu J. Emerging technologies in Si active photonics. J Semicond. (2018) 39:051001. doi: 10.1088/1674-4926/39/6/061001

6. Madelung O. (Ed.). Physics of Group IV Elements and III-V Compounds, Landolt-Börnstein: Numerical Data and Functional Relationships in Science and Technology, Vol. 17a. Berlin: Springer (1982).

7. Bauer M, Taraci J, Tolle J, Chizmeshya AVG, Zollner S, Smith DJ, et al. Ge-Sn semiconductors for band-gap and lattice engineering. Appl Phys Lett. (2002) 81:2992-94. doi: 10.1063/1.1515133

8. Mathews J, Beeler RT, Tolle J, Xu C, Roucka R, Kouvetakis J, et al. Direct-gap photoluminescence with tunable emission wavelength in $\mathrm{Ge}_{1-y} \mathrm{Sn}_{y}$ alloys on silicon. Appl Phys Lett. (2010) 97:221912. doi: 10.1063/1.3521391

9. Chen R, Lin H, Huo Y, Hitzman C, Kamins TI, Harris JS. Increased photoluminescence of strain-reduced, high-Sn composition $\mathrm{Ge}_{1-\mathrm{x}} \mathrm{Sn}_{\mathrm{X}}$ alloys grown by molecular beam epitaxy. Appl Phys Lett. (2011) 99:181125. doi: 10.1063/1.3658632

10. Li H, Brouillet J, Salas A, Wang X, Liu J. Low temperature growth of high crystallinity $\mathrm{GeSn}$ on amorphous layers for advanced optoelectronics. Opt Mater Express. (2013) 3:1385-96. doi: 10.1364/OME.3.001385

11. Bhargava N, Coppinger M, Prakash Gupta J, Wielunski L, Kolodzey J. Lattice constant and substitutional composition of $\mathrm{GeSn}$ alloys grown by molecular beam epitaxy. Appl Phys Lett. (2013) 103:041908. doi: 10.1063/1. 4816660 fabricated the GeSn materials and characterized their crystallinity and microstructures. AC and LJ fabricated and tested the photodetector devices with the help from SF. JP provided high purity $\mathrm{Sn}$ deposition source material. JL directed this research, analyzed the data with other co-authors, and coordinated the writing of this paper.

\section{FUNDING}

This work had been supported by the National Science Foundation under the grant number DMR-1255066. Additional support has been provided by Air Force Small Business Technology Transfer (STTR) program through prime contract \# FA8650-18-C-1638.

\section{ACKNOWLEDGMENTS}

The authors would to thank Prof. Christopher Levey at Thayer School of Engineering for supporting the rapid thermal annealing equipment and Drs. Charles Daghlian and Maxime Guinel at the Dartmouth Electron Microscopy Facility Center for supporting the electron microscopy training.

12. Oehme M, Kostecki K, Schmid M, Oliveira F, Kasper E, Schulze J. Epitaxial growth of strained and unstrained GeSn alloys up to $25 \% \mathrm{Sn}$. Thin Solid Films. (2014) 557:169-72. doi: 10.1016/j.tsf.2013.10.064

13. Ghetmiri SA, Du W, Margetis J, Mosleh A, Cousar L, Conley BR, et al. Directbandgap GeSn grown on silicon with $2230 \mathrm{~nm}$ photoluminescence. Appl Phys Lett. (2014) 105:151109. doi: 10.1063/1.4898597

14. Mathews J, Roucka R, Xie JQ, Yu SQ, Menéndez J, Kouvetakis J. Extended performance $\mathrm{GeSn} / \mathrm{Si}(100)$ p-i-n photodetectors for full spectral range telecommunication applications. Appl Phys Lett. (2009) 95:133506. doi: 10.1063/1.3238327

15. Gupta JP, Bhargava N, Kim S, Adam T, Kolodzey J. Infrared electroluminescence from $\mathrm{GeSn}$ heterojunction diodes grown by molecular beam epitaxy. Appl Phys Lett. (2013) 102:251117. doi: 10.1063/1.4812747

16. Du W, Zhou Y, Ghetmiri SA, Mosleh A, Conley R, Nazzal A, et al. Room-temperature electroluminescence from $\mathrm{Ge} / \mathrm{Ge}_{1-\mathrm{x}} \mathrm{Sn}_{\mathrm{X}} / \mathrm{Ge}$ diodes on $\mathrm{Si}$ substrates. Appl Phys Lett. (2014) 104:241110. doi: 10.1063/1.4884380

17. Wirths S, Geiger R, von den Driesch N, Mussler G, Stoica T, Mantl S, et al. Lasing in direct- bandgap GeSn alloy grown on Si. Nat Photonics. (2015) 9:88-92. doi: 10.1038/nphoton.2014.321

18. Cong H, Xue C, Zheng J, Yang F, Yu K, Liu Z, et al. Silicon based GeSn p-i-n photodetector for SWIR detection. IEEE Photonics J. (2016) 8:6804706. doi: 10.1109/JPHOT.2016.2607687

19. Stange D, Wirths S, Geiger R, Schulte-Braucks C, Marzban B, von den Driesch $\mathrm{N}$, et al. Optically pumped GeSn microdisk lasers on Si. ACS Photonics. (2016) 3:1279-85. doi: 10.1021/acsphotonics.6b00258

20. Al-Kabi S, Ghetmiri SA, Margetis J, Pham T, Zhou Y, Dou W, et al. An optically pumped $2.5 \mu \mathrm{m}$ GeSn laser on Si operating at $110 \mathrm{~K}$. Appl Phys Lett. (2016) 109:171105. doi: 10.1063/1.4966141

21. Li H, Wang X, Liu J. Highly effective strain-induced band-engineering of (111) oriented, direct-gap $\mathrm{GeSn}$ crystallized on amorphous $\mathrm{SiO}_{2}$ layers. Appl Phys Lett. (2016) 108:102101. doi: 10.1063/1.4943192

22. Margetis J, Al-Kabi S, Du W, Dou W, Zhou Y, Pham T, et al. Si-based GeSn lasers with wavelength coverage of $2-3 \mu \mathrm{m}$ and operating temperatures up to 180 K. ACS Photonics. (2017) 5:827-33. doi: 10.1021/acsphotonics.7b00938 
23. Reboud V, Gassenq A, Pauc N, Aubin J, Milord L, Thai QM, et al. Optically pumped GeSn micro-disks with $16 \%$ Sn lasing at $3.1 \mu \mathrm{m}$ up to $180 \mathrm{~K}$. Appl Phys Lett. (2017) 111:092101. doi: 10.1063/1.5000353

24. Zhou Y, Dou W, Du W, Ojo S, Tran H, Ghetmiri SA, et al. Optically pumped GeSn lasers operating at $270 \mathrm{~K}$ with broad waveguide structures on Si. ACS Photonics. (2019) 6:1434-41. doi: 10.1021/acsphotonics.9b00030

25. Beals M, Michel J, Liu J, Ahn D, Sparacin D, Sun R, et al. Process flow innovations for photonic device integration in CMOS. Proc SPIE. (2008) 6898:689804. doi: 10.1117/12.774576

26. Li H, Brouillet J, Salas A, Chaffin I, Wang X, Liu J. Low temperature geometrically confined growth of pseudo single crystalline $\mathrm{GeSn}$ on amorphous layers for advanced optoelectronics. ECS Trans. (2014) 64:81927. doi: 10.1149/06406.0819ecst

27. Li H, Brouillet J, Salas A, Chaffin I, Wang X, Liu J. Pseudo single crystal, direct-band-gap $\mathrm{Ge}_{0.89} \mathrm{Sn}_{0.11}$ on amorphous dielectric layers towards monolithic 3D photonic integration. Appl Phys Lett. (2014) 105:201107. doi: 10.1063/1.4902349

28. Li H, Cuervo Covian AV, Wang X, Liu J. High thermal stability of tensile strained direct gap GeSn crystallized on amorphous layers. ECS Trans. (2016) 75:623-32. doi: 10.1149/07508.0623ecst

29. Wang X, Li H, Liu J. Power-dependent transient gain study on direct gap GeSn crystallized on amorphous layers. ECS Trans. (2016) 75:2238. doi: 10.1149/07508.0223ecst

30. Corning's flexible Willow glass laminate (2019). Available online at: https://www.corning.com/worldwide/en/innovation/corning-emerginginnovations/corning-willow-glass.html (accessed August 20, 2019).

31. Thompson CV. Grain growth in thin films. Annu Rev Mater Sci. (1990) 20:245-68. doi: 10.1146/annurev.ms.20.080190.001333

32. Li H. Group IV semiconductors for Si photonics and solar energy applications (Ph.D. thesis). Dartmouth College, Hanover, NH, United States (2016).

33. Sun R, Beals M, Pomerene A, Cheng J, Hong CY, Kimerling LC, et al. Impedance matching vertical optical waveguide couplers for dense high index contrast circuits. Optics Express. (2008) 16:11682-90. doi: 10.1364/OE.16.011682

34. Liu J, Wang X. Epitaxy-free direct bandgap GeSn materials and devices for facile 3D photonic integration. In: Conference on Lasers and Electro-Optics. San Jose, CA: OSA Technical Digest (Optical Society of America, 2018), paper STh4I.1 (2018). doi: 10.1364/CLEO_SI.2018.STh4I.1

35. Cuervo Covian A, Zhou Y, Wang X, Yu SQ, Liu J. Sn Nanodots-induced composition enhancement (NICE) to achieve 26 at.\% Sn in GeSn for midinfrared integrated photonics. In: 2nd Joint ISTDM/ICSI 2019 Conference, 6/2-6/6, Madison, WI: University of Wisconsin-Madison (2019).

36. Burns G, Dill FH Jr, Nathan MI. The effect of temperature on the properties of GaAs laser. Proc IEEE. (1963) 51:947-8. doi: 10.1109/PROC.1963.2345

37. D'Costa VR, Cook CS, Birdwell AG, Littler CL, Canonico M, Zollner $S$, et al. Optical critical points of thin-film $\mathrm{Ge}_{1-\mathrm{y}} \mathrm{Sn}_{\mathrm{y}}$ alloys: a comparative $\mathrm{Ge}_{1-\mathrm{y}} \mathrm{Sn}_{\mathrm{y}} / \mathrm{Ge}_{1-\mathrm{x}} \mathrm{Si}_{\mathrm{x}}$ study. Phys Rev B. (2006) 73:125207. doi: 10.1103/PhysRevB.73.125207

38. Jiang L, Gallagher JD, Senaratne CL, Aoki T, Mathews J, Kouvetakis J, et al. Compositional dependence of the direct and indirect band gaps in $\mathrm{Ge}_{1-\mathrm{y}} \mathrm{Sn}_{\mathrm{y}}$ alloys from room temperature photoluminescence: implications for the indirect to direct gap crossover in intrinsic and n-type materials. Semicond Sci Technol. (2014) 29:115028. doi: 10.1088/0268-1242/29/11/ 115028

39. Polak MP, Scharoch P, Kudrawiec R. The electronic band structure of $\mathrm{Ge}_{1-\mathrm{x}} \mathrm{Sn}_{\mathrm{x}}$ in the full composition range: indirect, direct, and inverted gaps regimes, band offsets, and the Burstein-Moss effect. J Phys D Appl Phys. (2017) 50:195103. doi: 10.1088/1361-6463/aa67bf

40. Pezzoli F, Giorgioni A, Patchett D, Myronov M. Temperature-dependent photoluminescence characteristics of GeSn epitaxial layers. ACS Photonics. (2016) 3:2004-9. doi: 10.1021/acsphotonics.6b00438

41. Wang X, Kimerling LC, Michel J, Liu J. Large inherent optical gain from the direct gap transition of Ge thin films. Appl Phys Lett. (2013) 102:131116. doi: 10.1063/1.4800015

42. Xu X, Wang X, Nishida K, Takabayashi K, Sawano K, Shiraki Y, et al. Ultralarge transient optical gain from tensile-strained, n-doped germanium on silicon by spin-on dopant diffusion. Appl Phys Express. (2015) 8:092101. doi: 10.7567/APEX.8.092101
43. Bristow $\mathrm{AD}$, Rotenberg N, van Driel HM. Two-photon absorption and Kerr coefficients of silicon for $850-2200 \mathrm{~nm}$. App Phys Lett. (2007) 90:191104. doi: 10.1063/1.2737359

44. Mak G, van Driel HM. Femtosecond transmission spectroscopy at the direct band edge of germanium. Phys Rev B. (1994) 49:1681720. doi: 10.1103/PhysRevB.49.16817

45. Lange C, Köster NS, Chatterjee S, Sigg H, Chrastina D, Isella G, et al. Ultrafast nonlinear optical response of photoexcited $\mathrm{Ge} / \mathrm{SiGe}$ quantum wells: evidence for a femtosecond transient population inversion. Phys Rev B. (2009) 79:201306 (R). doi: 10.1103/PhysRevB.79.201306

46. Claussen SA, Tasyurek E, Roth JE, Miller DAB. Measurement and modeling of ultrafast carrier dynamics and transport in germanium/silicon-germanium quantum wells. Opt Express. (2010) 18:25596-607. doi: 10.1364/OE.18.025596

47. Chuang SL. Physics of Optoelectronic Devices. Volume 22 of Wiley Series in Pure and Applied Optics, New York, NY: Wiley (1995).

48. Werner J, Oehme M, Schmid M, Schirmer A, Kasper E, Schulze J. Germanium-tin p-i-n photodetectors integrated on silicon grown by molecular beam epitaxy. Appl Phys Lett. (2011) 98:061108. doi: 10.1063/1.3555439

49. Roucka R, Mathews J, Weng C, Beeler R, Tolle J, Menéndez J, et al. Highperformance near-IR photodiodes: a novel chemistry-based approach to $\mathrm{Ge}$ and Ge-Sn devices integrated on silicon. IEEE J Quantum Electron. (2011) 47:213-22. doi: 10.1109/JQE.2010.2077273

50. Su S, Cheng B, Xue C, Wang W, Cao Q, Xue H, et al. GeSn p-i-n photodetector for all telecommunication bands detection. Opt Express. (2011) 19:64005. doi: 10.1364/OE.19.006400

51. Oehme M, Schmid M, Kaschel M, Gollhofer M, Widmann D, Kasper E, et al. GeSn p-i-n detectors integrated on Si with up to 4\% Sn. Appl Phys Lett. (2012) 101:141110. doi: 10.1063/1.4757124

52. Oehme M, Kostecki K, Ye K, Bechler S, Ulbricht K, Schmid M, et al. GeSn-onSi normal incidence photodetectors with bandwidths more than $40 \mathrm{GHz}$. Opt Express. (2014) 22:839-46. doi: 10.1364/OE.22.000839

53. Pham T, Du W, Tran H, Margetis J, Tolle J, Sun G, et al. Systematic study of Si-based GeSn photodiodes with $2.6 \mu \mathrm{m}$ detector cutoff for short-wave infrared detection. Opt Express. (2016) 24:4519-31. doi: 10.1364/OE.24.0 04519

54. Gassenq A, Gencarelli F, Van Campenhout J, Shimura Y, Loo R, Narcy $\mathrm{G}$, et al. GeSn/Ge heteostructure short-wave infrared photodetectors on Silicon. Opt Express. (2012) 20:27297-303. doi: 10.1364/OE.20.0 27297

55. Dong Y, Wang W, Xu S, Lei D, Gong X, Guo X, et al. Two-micro-wavelength germanium-tin photodiodes with low dark current and gigahertz bandwidth. Opt Express. (2017) 25:15818-27. doi: 10.1364/OE.25.015818

56. Peng YH, Cheng HH, Mashanov VI, Chang GE. GeSn p-i-n waveguide photodetectors on silicon substrates. Appl Phys Lett. (2014) 105:231109. doi: 10.1063/1.4903881

57. Liu J, Cannon DD, Wada K, Ishikawa Y, Jongthammanurak S, Danielson DT, et al. Tensile strained Ge p-i-n photodetectors on $\mathrm{Si}$ platform for C and L band telecommunications. Appl Phys Lett. (2005) 87:011110. doi: 10.1063/1.1993749

58. Thorlabs (2019). Available online at: https://www.thorlabs.com/drawings/ 674eb64b187d9912-43AED979-5056-0103-7954CD792A847D84/FDPS3X3Manual.pdf

Conflict of Interest: JP is the founder of Epitaxial Laboratory Inc. and a Research Affiliate at Dartmouth College.

The remaining authors declare that the research was conducted in the absence of any commercial or financial relationships that could be construed as a potential conflict of interest.

Copyright (C) 2019 Wang, Cuervo Covian, Je, Fu, Li, Piao and Liu. This is an openaccess article distributed under the terms of the Creative Commons Attribution License (CC BY). The use, distribution or reproduction in other forums is permitted, provided the original author(s) and the copyright owner(s) are credited and that the original publication in this journal is cited, in accordance with accepted academic practice. No use, distribution or reproduction is permitted which does not comply with these terms. 